\title{
Ultraluminous Infrared Galaxies
}

\author{
Carol J. Lonsdale, Duncan Farrah \& Harding E. Smith
}

Summary. Ever since their discovery in the 1970's, UltraLuminous InfraRed Galaxies (ULIRGs; classically $L_{i r}>10^{12} L_{\odot}$ ) have fascinated astronomers with their immense luminosities, and frustrated them due to their singularly opaque nature, almost in equal measure. Over the last decade, however, comprehensive observations from the $\mathrm{X}$-ray through to the radio have produced a consensus picture of local ULIRGs, showing that they are mergers between gas rich galaxies, where the interaction triggers some combination of dust-enshrouded starburst and AGN activity, with the starburst usually dominating. Very recent results have thrown ULIRGs even further to the fore. Originally they were thought of as little more than a local oddity, but the latest IR surveys have shown that ULIRGs are vastly more numerous at high redshift, and tantalizing suggestions of physical differences between high and low redshift ULIRGs hint at differences in their formation modes and local environment. In this review we look at recent progress on understanding the physics and evolution of local ULIRGs, the contribution of high redshift ULIRGs to the cosmic infrared background and the global history of star formation, and the role of ULIRGs as diagnostics of the formation of massive galaxies and large-scale structures.

\section{Introduction}

\subsection{The Biggest and the Brightest}

In human endeavour there's a fascination with the "biggest and the best", or the "best and the brightest". It's a matter for the social and psychological sciences to speculate on the reasons we feel driven to give Oscars to the best movies and to climb the highest mountains, but being human (well, most of them anyway), astrophysicists are not immune to the desire to search for the Universe's own brand of the biggest and the brightest. We tend to give them extreme names such as ultra-this or hyper-that. In their rarity however, unusual objects and environments teach us about the most extreme physical processes in the Universe.

Which brings us to ULIRGs (or ULIGs to some) - Ultra Luminous InfraRed Galaxies. There are also HLIRGs (or HiLIRGs or HyLIRGs, or indeed HyLIGs): Hyper Luminous InfraRed Galaxies. These Oscar contenders have historically 
been defined simply in terms of luminosity: $L_{8-1000 \mu m}$ between $10^{12}$ and $10^{13} L_{\odot}$ for the ULIRGs and $>10^{13} L_{\odot}$ for HLIRGs ${ }^{1}$. What are these dramatic objects, and why are they among the brightest objects in the Universe? Does this also imply they are the biggest in term of mass or size, or are they just superficial fireworks that leave little lasting impression? What can understanding these enigmatic objects teach us about the evolution of galaxies and how the the Universe came to look as it now does?

The last few years have seen a dramatic shift in our perceptions of ULIRGs. Once believed by many to be a rare curiosity - certainly interesting, yes, but perhaps no more than a local oddity - they're beginning to see center stage much more frequently. Ironically, this increased interest in these rare objects has arisen because we now realize that ULIRGs were once not nearly so rare as we find them to be in the local Universe: pioneering submillimeter and millimeter surveys have demonstrated that ULIRGs are many hundreds of times more numerous at z $>1$ than they are locally. This in turn suggests that they played a much more important role in galaxy formation and evolution than we imagined, and so understanding them becomes of prime importance. Fortunately, this rise in the fortunes of the ULIRG has occurred in an era when many new observing capabilities are coming on line. Foremost among these is the Spitzer Space Telescope, with its suite of deep imaging infrared cameras and its sensitive infrared spectrograph. Spitzer's extensive first results on ULIRGs are just now beginning to be published. Submillimeter and millimeter cameras are also improving dramatically; upcoming facilities include AzTEC, SCUBA-2, Herschel, and ALMA. We can also look forward to major new insights from mid- and near-IR facilities such as ASTRO-F, WISE, and JWST, as well as radio facilities such as the Square Kilometer Array, and new X-ray facilities with high hard X-ray sensitivities such as Con-X and XEUS. The timeliness of this subject is exemplified by recent reviews of The Cosmic InfraRed Background (Lagache. Puget \& Dole, 2005), Interacting Galaxies (van Gorkum \& Hibbard, 2005). Megamasers (Lo, 2005), Galactic Winds (Veilleux. Cecil. \& Bland-Hawthorn, 2005), and High Redshift Molecular Gas (Vanden Bout \& Solomon, 2005), all of relevance to ULIRGs.

\subsection{Overview}

ULIRGs were first discovered in large numbers by the Infrared Astronomical Satellite in 1983, and were found to be comparatively rare locally, with a space density several orders of magnitude lower than that of normal galaxies, and possibly a factor of a few higher than QSOs. Followup observations show that most, if not all ULIRGs are found in major disk mergers, and that the central few hundred pc of their nuclear regions harbour very large masses of gas and dust. The power source behind the IR emission is some combination of a large popu-

\footnotetext{
${ }^{1}$ The supporting cast consists of LIRGs (or LIGs), the much more common lower luminosity understudies of the prima donnas, with $L_{8-1000 \mu m}$ between $10^{11}$ and $10^{12} L_{\odot}$
} 
lation of hot young stars (a 'starburst'") or a very massive black hole accreting matter at a rapid rate (which for the remainder of this review we refer to as an 'AGN'). Though distinguishing between the two initially (and even now) proved to be very difficult, it is now thought that, at least locally, ULIRGs are mainly powered by a starburst, but frequently with a significant AGN contribution. Local ULIRGs reside in relatively low-density environments (not unexpectedly, since relative velocities are thought to be too high for mergers to occur in rich, virialized environments), and are expected to evolve into spheroidal systems as the galaxy mergers that appear to trigger ULIRG activity progresses.

Even IRAS was sensitive enough to determine that there has been very strong evolution in the ULIRG (and LIRG) population with redshift out to at least $z \sim 0.5$, with approximate form $(1+z)^{4}$. IRAS also found ULIRGs out to extremely high redshifts, including the famous, lensed, F10214+4724 at $\mathrm{z}=2.286$. This strong evolution was confirmed with results from the Infrared Space Observatory which, although covering much smaller areas than IRAS, could probe this evolution out to $z \sim 1$ due to its greater sensitivity (Figure 1). This evolution was later recast as the now ubiquitous 'star formation history of the Universe' figures, which show that LIRGs rather than ULIRGs are responsible for the bulk of the evolution seen since $\mathrm{z} \sim 1$ in IR galaxies. ULIRGs, however, did not slink into the shadows; on the contrary they returned triumphant with the advent of submillimeter imaging surveys, which came shortly after ISO and can in principle probe IR-luminous galaxies up to $z \sim 7$. These sub-mm surveys showed that ULIRGs are orders of magnitude more numerous at $z>1$ than locally, outnumbering optically bright QSOs at those redshifts by a large margin. Followup observations showed that these distant ULIRGs bear many similarities to their local cousins, but also exhibit some key differences, and that they may signpost the obscured phases of the very dramatic events suspected of building the most massive galaxies seen in the local Universe.

When considered within the framework of modern theories for the formation of galaxies and large-scale structure, it seems initially surprising that there are many more ULIRGs at high redshift than locally, because in early implementations of the 'hierarchical buildup' paradigm, large galaxies build up slowly from the mergers of smaller systems. This is in contrast to the early 'monolithic collapse' models (Eggen. Lvnden-Bell \& Sandage, 1962), where ellipticals formed early in a dramatic burst of star formation, which had been largely supplanted in favour of hierarchical models. The discovery of so many ULIRGs at high redshifts caused hierarchical models of the time major difficulties in making enough distant systems with such high star formation rates. The basic dark matter halo growth theory, described by an extended/modified Press-Schechter formalism, does however allow for rapid baryon accumulation in very massive dark matter halos, and recent galaxy formation models are having greater success in producing the observed number of ULIRGs in sub-mm surveys, albeit with some stringent assumptions.

\footnotetext{
${ }^{2}$ for our purposes defined as a star forming event with a gas exhaustion timescale very short compared to the Hubble time
} 
In this review, we will therefore focus on selected key topics: (1) our understanding of the astrophysics of local ULIRGs, and in particular the relative importance of star formation versus AGN in powering ULIRGs, (2) similarities and differences between local and high-redshift ULIRGs, and (3) the relationship between ULIRGs and the formation of large-scale structure and of galaxies as a function of redshift. Since the study of ULIRGs clearly connects to many major disciplines of observational and theoretical extragalactic astronomy we cannot hope to cover all topics of relevance to them in this review. Nor can we completely review all recent published studies of ULIRGs; excellent ULIRG papers simply abound. We therefore highlight the most recent advances and our perspective on the most important questions concerning their study within the framework of galaxy and structure formation.

In section 2 we provide brief historical context to the discovery of ULIRGs and their evolutionary role. In section 3 we review current understanding of the astrophysics of local ULIRGs by wavelength, and in section 4 we summarize local studies into a picture of ULIRG nature and evolution in the local Universe. In section 5 we review observations of ULIRGs at higher redshifts, based primarily on data from ISO, SCUBA, HST and Spitzer. Section [6 places these studies into the context of structure formation and reviews their role within galaxy formation scenarios. Finally, section 7 highlights the key open questions and our perspectives of where the answers are likely to come from.

For the remainder of this review we usually refer to ULIRGs and HLIRGs combined as ULIRGs, because many of the earlier works used the term "ULIRG" to refer to all objects above $10^{12}$ in $L_{\odot}$ and the term HLIRG has been in only recent and inconsistent use. We assume $H_{0}=70 \mathrm{~km} \mathrm{~s}^{-1} \mathrm{Mpc}^{-1}, \Omega=1$, and $\Omega_{\Lambda}=0.7$. Luminosities are quoted in units of bolometric solar luminosities, where $L_{\odot}=3.826 \times 10^{26}$ Watts. Unless otherwise stated, the term 'IR' or 'infrared' luminosity refers to the integrated rest-frame luminosity over 1-1000 or 8-1000 $\mu \mathrm{m}$ (which differ very little for most SEDs).

\section{The discovery of ULIRGs}

It is often stated that UltraLuminous InfraRed Galaxies were discovered by the InfraRed Astronomy Satellite (IRAS), which was launched in 1983. Strictly speaking this may be true since the first objects to meet the now generally acknowledged criteria which define a ULIRG $-L_{8-1000 \mu m}>10^{12} \mathrm{~L}_{\odot}$ and, optionally, exceptionally large ratios of $L_{\text {infrared }} / L_{\text {optical }}$ - can indeed be considered to have been discovered with the publication by Houck et al. (1985) of a sample of 9 IRAS sources invisible or exceptionally faint on the Palomar Sky Survey plates, and exhibiting $L_{i r} / L_{\text {opt }}$ ratios over 50 . We must go back further, however, to appreciate the origins of the ULIRG phenomenon. Galaxies with an unusually high amount of mid- or far-infrared emission compared to their optical output had been discovered by the pioneers of infrared astronomy in the 10 to 15 years preceding IRAS, and had been recognised to represent luminous, probably short-lived, active events of some sort within a galaxy. 

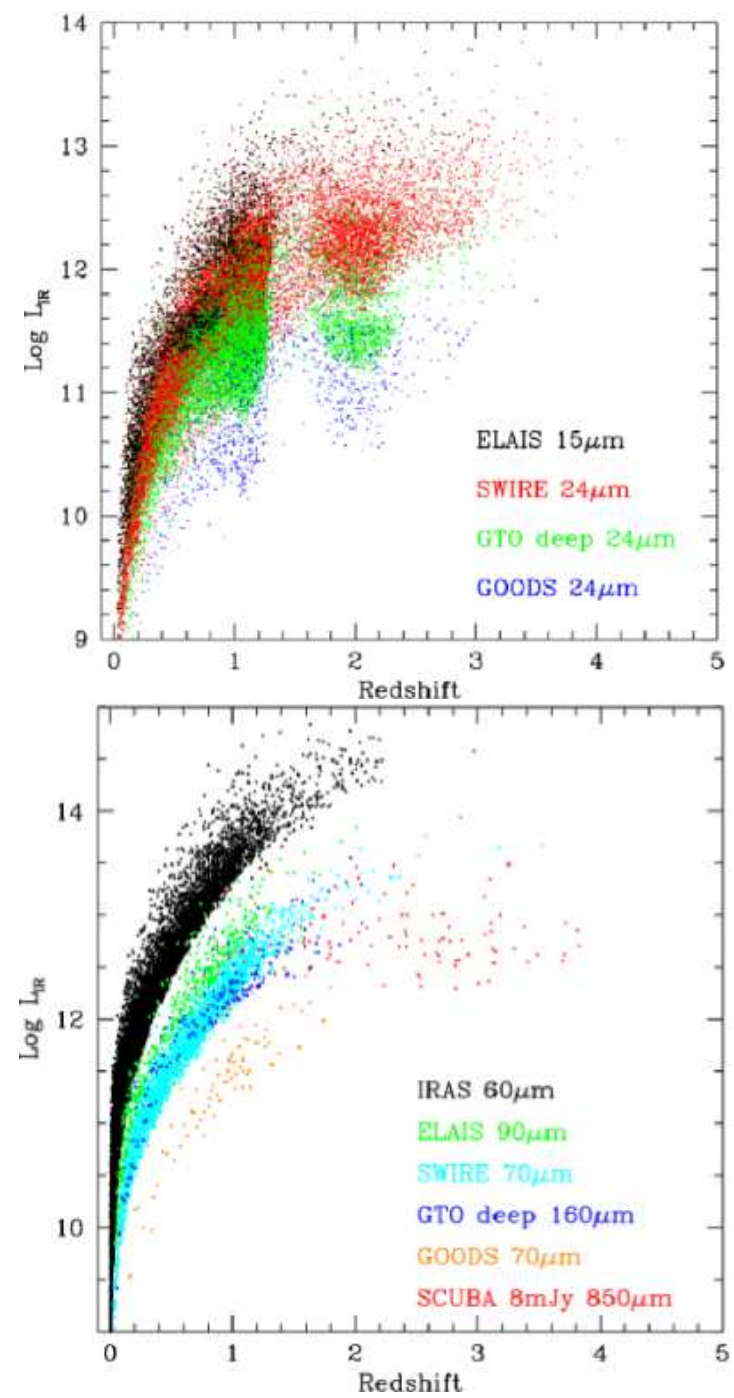

Fig. 1. Simulation of the ability of recent infrared surveys to discover ULIRGs, with shorter wavelength $(\lambda<25 \mu \mathrm{m})$ surveys plotted in the upper panel and longer wavelength surveys below. The band in the upper figure at $z \sim 1.5$ is produced by the the $10 \mu \mathrm{m}$ silicate absorption feature which falls in the Spitzer $24 \mu \mathrm{m}$ band at that redshift. Wide shallow surveys have the largest volume for discovering the most luminous ULIRGs, while narrow deep surveys can of course find the most distant ones, though in much smaller number. Tiered ('wedding cake') surveys are thus required to construct complete luminosity functions within a given redshift interval (vertical slices). Based on the simulations of $\mathrm{Xu}$ et al. (2003), these simulations can fit IRAS, ISO, Spitzer and submillimeter counts and redshift distributions. We plot $1 / 5$ of the objects expected within the area and depth of each survey: ISO ELAIS $15 \mu \mathrm{m}$ (Vaccari et al., 2005); SWIRE 24 \& 70 $\mathrm{m}$ (Surace et al., 2005); Spitzer Guaranteed Time Observer

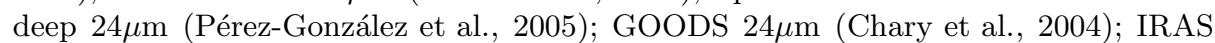
$60 \mu \mathrm{m}$ (Lonsdale et al., 1990); SCUBA $850 \mu \mathrm{m}$ (Scott et al. 2002); GOODS $70 \mu \mathrm{m}$ (D. Frayer, priv. comm.); Spitzer GTO deep 160 $\mu$ m (Dole et al., 2004). 
Several excellent reviews have described the IRAS and pre-IRAS legacy, including Rieke and Lebofsky (1979), Soifer et al. (1987), Sanders \& Mirabel (1996), and Mirabel (2002) so we present here only the brief highlights relevant to ULIRG discovery and ULIRG samples.

The first infrared observations of galaxies were undertaken in the late sixties (Low \& Kleinmann, 1968; Kleinmann \& Low, 1970), and a population of galaxies with infrared-dominant emission from the nuclear regions was discussed by Rieke \& Low (1972). The well known radio-infared relation (de Jong et al., 1985; Helou et al., 1985) was originally suggested by van der Kruit (1971). An early debate ensued as to the thermal vs non-thermal origin of the infrared emission from galaxies (Rees et al., 1969; Burbidge \& Stein, 1970) with the conclusion that most systems are powered by re-radiation of starlight by dust (Rieke \& Lebofsky 1979 and references therein). The other key contribution of the preIRAS days was the role of interactions in triggering nuclear and starburst activity (Toomre \& Toomre, 1972; Larson \& Tinslev, 1978), which was confirmed for the strongly interacting system Arp 299 by Gehrz et al. (1983), and several other interacting and merging systems by Lonsdale. Persson \& Matthews (1984); Joseph \& Wright (1985).

IRAS scanned almost the entire sky in the thermal infrared ${ }^{3}$, observing in four bands centered at 12, 25, 60 and $100 \mu \mathrm{m}$ and opening up an unprecedented volume of space to study in this wavelength regime. This allowed IRAS to find exceedingly rare objects and to demonstrate the importance of this new class of exceptionally infrared-luminous objects (Figure 1). One of the most exciting early discoveries was that IRAS would not be limited to exploring only the very local Universe due to its relatively bright flux limits, but it could reach out to significant distances thanks to the existence of large numbers of exceptionally infrared-luminous sources. Strong evolution was demonstrated for the IRAS deep north ecliptic hole field by Hacking. Houck \& Condon (1987) and confirmed over the sky by Lonsdale et al. (1990) and Saunders et al. (1990). Extensive redshift surveys (Oliver et al., 1996) led to the discovery of the first $\mathrm{z}>2$ IRAS HLIRG, IRAS FSC $10214+4724$ at $\mathrm{z}=2.86$ (Rowan-Robinson et al., 1991) which is a lensed system. The extremely high evolution rates, modeled, for example, as $L_{I R} \sim(1+z)^{\sim 4}$, were actually anticipated, based on the previously known strong evolution of starburst-related sub-mJy radio sources (Hacking. Houck \& Condon, 1987).

ULIRGs were found to have comparable space densities to those of PG QSOs of similar luminosity by Soifer et al. (1987); however, significant incompleteness in the PG QSO sample has since been demonstrated (Wisotzki et al., 2000) so this result needs re-visiting. The best known samples of IRAS luminous and ultraluminous galaxies, all selected at $60 \mu \mathrm{m}$, are the Bright Galaxy Sample of Soifer et al. (1987), recently significantly updated into the Revised Bright

\footnotetext{
${ }^{3}$ defined as the wavelength region over which dust grains can thermally re-radiate emission, ranging from the dust sublimation temperature on the short wavelength side $(\sim 1 \mu \mathrm{m})$ to $\sim 1000 \mu \mathrm{m}$ on the long wavelength side where non-thermal processes often begin to dominate
} 
Galaxy Sample (RBGS; Sanders et al. 2005), and the complete flux-limited IRAS 1 Jy sample (Kim \& Sanders, 1998). Also notable are the 2Jy sample of Strauss et al. (1990) and the FIRST/IRAS sample of Stanford et al. (2000). The RBGS contains 629 IRAS $60 \mu \mathrm{m}$ galaxies brighter than 5.24 Jy and Galactic latitude $>5$ degrees and contains 20 ULIRGs; the most luminous being Mrk 231 with $L_{i r}=3.2 \times 10^{12} L_{\odot}$, the highest redshift being IRAS 07251-0248 at $\mathrm{z}=0.0876$, and the closest being Arp 220 at $\mathrm{z}=0.018$. The 1 Jy sample consists of 118 ULIRGs drawn from the IRAS Faint Source Catalog, with declination >40 degrees and Galactic latitude $|b|>30$ degrees. This sample also has a warm $60 / 100 \mu \mathrm{m}>0.3$ colour selection, which introduces a bias against cooler objects.

Whilst dramatic in nature, LIRGs and ULIRGs in the local Universe are rare, and they contribute only $\sim 6 \%$ of the total infrared luminous energy density (Soifer \& Neugebauer, 1991), and about $\sim 3 \%$ of the total energy density. It was found that both the IRAS $60 / 100 \mu \mathrm{m}$ colour and the $L_{I R} / L_{o p t}$ ratio increased with luminosity (Soifer et al., 1987), reaching $\sim 100$ in the most extreme systems. This indicates that the more luminous systems must have an increasing contribution from an additional warm source compared to the relatively cool emission from modest levels of star formation seen in spiral disks (de Jong et al., 1985). IRAS ULIRGs have been separated into warm and cool subsamples based on the 25/60 and 60/100 $\mu \mathrm{m}$ IRAS colours (Sanders et al., 1988b; Surace et al., 1998), with the warmer objects more likely to host an AGN (de Griip et al., 1985). The spectral energy distributions of IRAS ULIRGS were reviewed by Sanders and Mirabel (1996), illustrating these trends with colour and luminosity. These reviewers also compared the SEDs of QSOs and Blazars with those of ULIRGs.

It should be remembered that although the IRAS datasets are now nearly a quarter of a century old, they are very under-explored. As of publication date there are >37,000 infrared-bright galaxies in the IRAS Faint Source Catalog (FSC) that have never been observed with any other instrument and reported in any journal article. Only $43 \%$ of the 64,606 IRAS extragalactic FSC sources ${ }^{4}$ have been included in any sort of publication (J. Mazzarella, priv. comm.).

\section{The Physics of Local ULIRGs}

The complexity of the ULIRG phenomenon requires a multi-wavelength approach and we have learned an enormous amount from detailed studies at many wavelengths. For this review we focus on those observational areas which we believe are key to understanding the ULIRG phenomenon, in part because they represent the spectral regions of lowest optical depth, and in which key advances have been made in the years since the Sanders \& Mirabel review. We stress, however, that even the relatively low-optical depth radio, mid-infrared, and X-ray regimes may still show substantial obscuration due to starburst-related free-free absorption (Condon et al., 1991), compact nuclear molecular clouds or molecular

\footnotetext{
${ }^{4}$ As reported by NED, the NASA/IPAC Extragalactic Database
} 
tori, which may be optically thick at wavelengths as long as $30 \mu \mathrm{m}$, or Comptonthick absorbing columns with $N_{H} \gtrsim 10^{24} \mathrm{~cm}^{-2}$.

The compactness of the IR-emitting regions in luminous IR galaxies (c.f. Condon et al. 1991) suggests two possible origins for the high ULIRG luminosities: compact nuclear starbursts and/or highly obscured AGN activity. Much early effort was given to determining which phenomenon powers ULIRGs; with the understanding that starburst and AGN emission are frequently found together in luminous galaxies, the emphasis has shifted to determining which mechanism is dominant and to understanding the relationship between co-existing AGN \& starburst emission.

\subsection{Optical to mid-IR Imaging}

Early imaging surveys of IR-luminous sources spanned a wide range in luminosity (from $<10^{9} L_{\odot}$ up to $\sim 10^{12} L_{\odot}$, and revealed an interesting picture (Soifer et al., 1984; Rieke \& Lebofskv, 1986). Sources with $L_{i r}<10^{9} L_{\odot}$ are almost exclusively confined to undisturbed E and S0 systems, with few spirals. The fraction of spirals increases sharply with increasing IR luminosity however, with most systems in the range $10^{10} L_{\odot}<L_{i r}<10^{11} L_{\odot}$ being $\mathrm{Sb}$ or Sc type systems. At luminosities above about $10^{11} L_{\odot}$, the majority of systems are still spirals, but an increasing fraction (up to $\sim 25 \%$ ) appear to be involved in interactions, or to show signs of morphological disturbance.

This apparent increase in the number of interacting IRAS systems was thrown into sharp relief by early ULIRG imaging studies, showing that interactions and mergers are much more common amongst ULIRGs than in lower luminosity systems, though the exact fraction in ongoing interactions remained contentious for some years. The first imaging surveys (Armus. Heckman \& Milev, 1987) showed that at least $70 \%$ of systems with ULIRG or near-ULIRG luminosities are interacting, with morphologies expected from the collision of two disk galaxies. Later optical and near-IR studies of ULIRGs (Melnick \& Mirabel, 1990; Hutchings \& Neff, 1991; Clements et al., 1996) found a higher fraction of ULIRGs involved in interactions, at least $90 \%$, and that there are a wide range of merger stages present in the ULIRG population, from widely separated systems to advanced mergers (Murphy et al., 1996). Other studies, however, found a much lower fraction of ULIRGs involved in interactions, fewer than 70\% (Lawrence et al., 1989; Zou et al., 1991; Leech et al., 1994).

These results demonstrate that interactions and mergers play an important role in the ULIRG population. This provided a plausible trigger for the immense IR luminosities seen in ULIRGs. In order to both fuel and enshroud the power sources in ULIRGs (irrespective of whether they are starbursts, AGN, or both), a large quantity of gas and dust needs to be channeled into a small volume, most plausibly sited in the nucleus of the host galaxy. Results from N-body modeling of galaxy collisions (see Barnes \& Hernquist 1992 for a review) suggest that this is readily achieved during the course of a merger (Barnes, 1989; Barnes \& Hernquist, 1996; Mihos \& Hernquist, 1996: Dubinski. Mihos \& Hernquist, 1999), though details depend on many variables 
(e.g. angle of approach, relative velocity, disc inclinations, bulge size and gas and dark matter masses). The majority of mergers involve an initial close approach, followed by a maximum separation of up to $\sim 50 \mathrm{kpc}$, reached after $\sim 2.5 \times 10^{8}$ years, then a second close approach a few tens of millions of years later, which is rapidly followed by coalescence, and relaxation towards an elliptical profile. Total times scales to coalescence range from $\sim 7 \times 10^{8}$ years to $\sim 2 \times 10^{9}$ years, depending on the parameters of the encounter. Gas and dust can be channeled into the nuclear regions of the progenitors in one of two ways; either before coalescence, when tidal forces during the first close approach form bars in one or both progenitors (particularly if the progenitors have small bulge components) which are very efficient at channeling material into the central regions of a galaxy, or during coalescence, when shocks drive very large quantities of gas and dust into the nuclear regions. More recent N-body simulations have in general supported these conclusions, but added an intriguing possibility; under certain conditions (particularly if the progenitors are gas dominated), a merger between two disk galaxies can result in a disklike remnant, rather than an elliptical (Naab \& Burkert, 2003; Springel \& Hernquist, 2005; Robertson et al., 2005). It seemed therefore that galaxy mergers could provide all the necessary physical conditions for triggering a ULIRG, and that this implicated ULIRGs in the formation of elliptical galaxies. Furthermore, these simulations could explain a result that had been puzzling; that ULIRG activity could apparently be triggered in mergers when the progenitors are still physically separate.

Optical imaging from the Hubble Space Telescope (HST) offered enhanced resolution and sensitivity over ground based facilities. An early HST study of ULIRGs using WFPC2 (Surace et al., 1998) focused on a small sample with 'warm' infrared colours ${ }^{5}$, which biases towards systems likely to contain an obscured AGN. All of the sample were found to be interacting, with complex structures in their nuclear regions. Several systems showed a large number of compact bright 'knots' a few hundred pc in diameter, whose ages suggest they result from the merger. A complementary ground-based survey of ULIRGs with 'cool' IR colours (Surace et al., 2000) found a similar picture; all the galaxies show signs of interactions, from early to late stage, and many systems harbour 'knots' similar to those seen in the warm sample. The optical magnitudes are in most cases relatively modest (at least compared to the enormous IR luminosities); most systems have $\sim L^{*}$ luminosities, with very few being substantially brighter. A later HST survey of a larger, unbiased ULIRG sample (Farrah et al., 2001) found similar results; nearly all of the sample are interacting, with a wide range of merger stages. A small number host optical QSOs whose host galaxies are either interacting, or elliptical-like. Other authors have, on the basis of HST data, suggested that some ULIRGs show evidence for mergers between more than two galaxies, suggesting that ULIRGs may be the remnants of compact galaxy groups (Borne et al., 2000). HST imaging of ULIRGs in the near-infrared with NICMOS (Colina et al., 2001; Bushouse et al., 2002) has produced very similar

\footnotetext{
${ }^{5}$ i.e systems with $f_{25} / f_{60}>0.2$, where $f_{25}$ and $f_{60}$ are the $25 \mu \mathrm{m}$ and $60 \mu \mathrm{m}$ IRAS fluxes respectively
} 


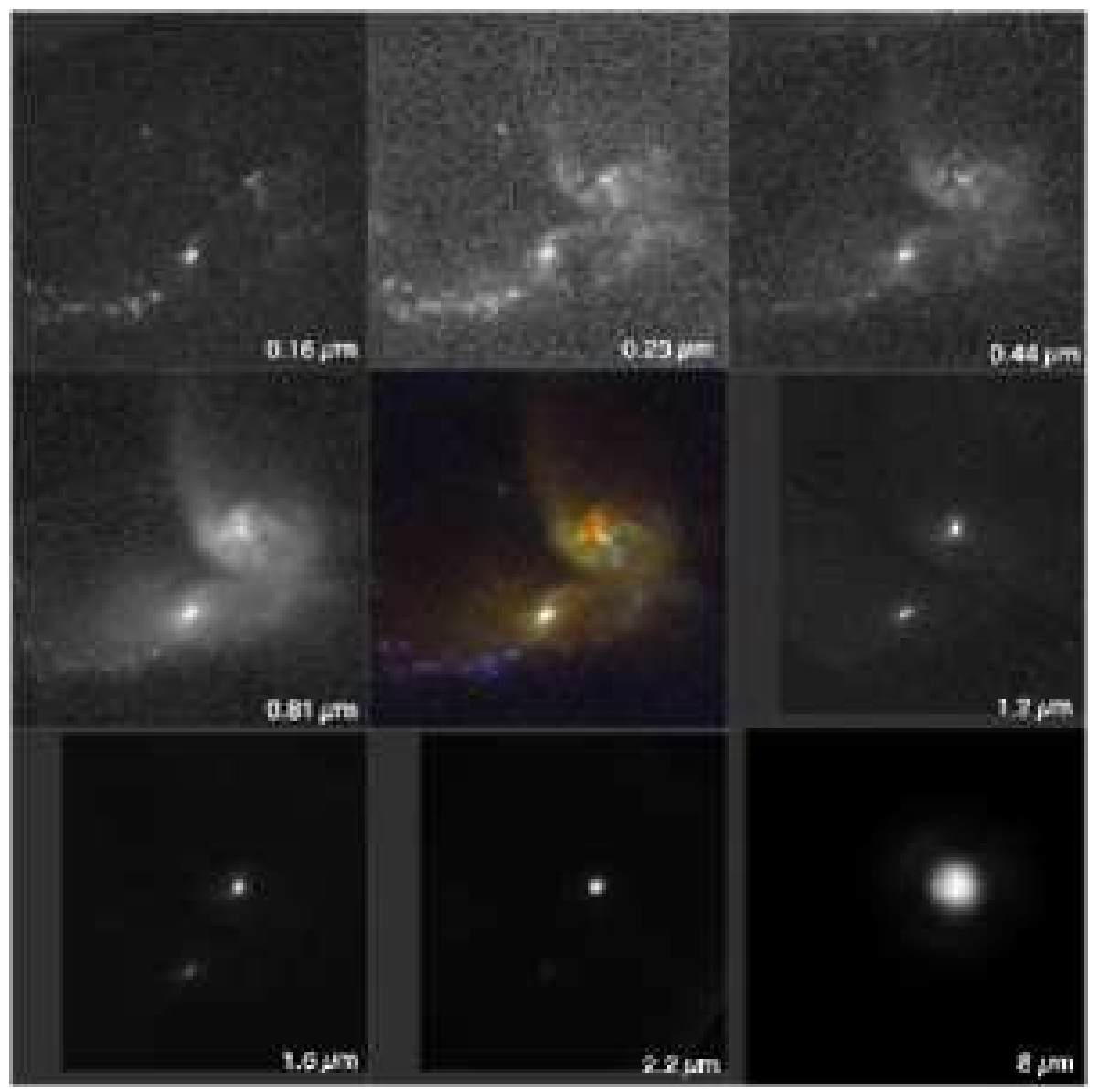

Fig. 2. IRAS $08572+3915$, as seen at many wavelengths. The colour composite at the center encodes wavelengths from the far-UV to the near-IR as blue-to-red, and illustrates the complex composite nature of ULIRG systems. The ultraviolet emission is dominated by one of the two merger nuclei and by young super-star clusters along the leading edge of one of the tidal tails. Almost no ultraviolet or blue emission is seen from the NW nucleus. At optical wavelengths, increasingly dominated by old stars, we see the merger galaxy body, which appears to be two spirals, accompanied by tidal tails. The NW nucleus has a complex structure of dust lanes. As we proceed into the thermal infrared, all emission sources other than a single compact emission source in the NW nucleus fade away. The images from $0.16-2.2 \mu \mathrm{m}$ are from STIS, WFPC2, and NICMOS (Goldader et al., 2002; Surace et al., 1998, 2000; Scoville et al., 2000). The $8 \mu \mathrm{m}$ image is from Spitzer (Surace et al, in prep), and has a beam size $\sim 5$ times greater than the HST data, but is sensitive enough to exclude much emission from the SE nucleus. Keck observations (Soifer et al., 2000) at similar wavelengths constrain the size of the nucleus to $<0.3$ arcseconds, or 300 pc. (J. Surace, priv. comm.). 
results to those from optical imaging, with at least $90 \%$ being interactions between two or (possibly) more progenitors over a wide range of merger stages, and that very few are much brighter than $L^{*}$.

To date however, the largest optical/NIR imaging survey of ULIRGs has been done from the ground; Veilleux. Kim. \& Sanders (2002) present R and K images for 118 ULIRGs from the IRAS 1Jy spectroscopic survey, and find that virtually all show signs of interactions, though very few showed definite signs of interactions between more than two progenitors. Most of the systems appear to be late-stage mergers, especially for the more luminous systems and/or those with spectroscopic signatures of an AGN, and typically have luminosities (in the $\mathrm{K}$ band) of $L^{*}$ or greater. The most advanced mergers show evidence for emerging elliptical profiles.

Near diffraction-limited Keck mid-infrared imaging of ULIRGs has been obtained by Soifer et al. $(1999 ; 2000)$ who find extremely compact structures, with spatial scales smaller than $0^{\prime \prime} .3$ in six of the seven ULIRGs observed. These compact sources emit between $30 \%-100 \%$ of the mid-infrared energy from these galaxies. In Mrk 231, IRAS 05189-2524 and IRAS $08572+3915$ there is strong evidence that the source size increases with increasing wavelength, suggesting heating by a central rather than extended source, consistent with the optical classification as an AGN. Spitzer mid-IR imaging programs of over 200 nearby ULIRGs (ongoing programs of J. Surace (Fig. 2) and J. Mazzarella) will provide great sensitivity in this important wavelength region, if limited spatial resolution.

\subsection{Optical \& near-IR Spectroscopy}

It might be suspected that spectroscopy at wavelengths shortward of a few microns would be useful mainly for redshift surveys, given the dust-enshrouded nature of ULIRGs. This, however, is a misconception; optical spectroscopic signatures of starburst and AGN activity are still apparent even when such activity is moderately obscured (up to and surpassing $A_{V} \sim 10$ depending on the observations), and starbursts and AGN can be detected in polarized light even when the direct line of sight is completely hidden. Furthermore, spectroscopic diagnostics from the UV through to the near-IR are, on the whole, more mature than those at longer wavelengths (Baldwin. Phillips \& Terlevich, 1981; Veilleux \& Osterbrock, 1987; Osterbrock. Tran \& Veilleux, 1992; Dopita et al., 2000), and line strengths, ratios and profile shapes can provide powerful constraints on the nature of the source of excitation. Recent advanced spectral synthesis codes (e.g. Leitherer et al., 1999; Kewley et al., 2001) allow for insightful diagnostics of starburst events, particularly in the UV where direct emission from hot young star photospheres, rather than reprocessed light from dust, is being sampled.

Early optical spectroscopic surveys of ULIRGs generally showed that they were mostly starburst-like in the optical (Elston. Cornell \& Lebofskv, 1985), while samples with 'warmer' infrared colours appeared more biased towards Seyferts or LINERS (de Griip et al., 1985; Osterbrock \& de Robertis, 1985), or 
even optical QSOs (Beichman et al., 1986). Conversely, studies of ULIRGs with 'cool' IR colours generally indicated the presence of starbursts (Heckman. Armus \& Milev, 1987; Armus. Heckman \& Milev, 1988), sometimes accompanied by spectral signatures of Wolf-Rayet stars, indicating that the starburst was likely only a few Myr old. Also noted at this time was a tendency for more IR-luminous objects to exhibit Seyfert spectra, with narrow lines in direct light (Cutri et al., 1994) and broad lines in polarized light (e.g. Hines et al., 1995).

To gain a complete picture of the spectroscopic properties of ULIRGs, however, requires large-scale surveys, which were soon forthcoming as part of IRAS followup. One of the largest to date is the IRAS 1Jy survey (Kim. Veilleux \& Sanders, 1998; Veilleux. Kim \& Sanders, 1999a), which showed that the majority of ULIRGs have optical spectra reminiscent of starbursts, but with a systematic increase in the fraction of ULIRGs with Seyfert (1 or 2) spectra with increasing IR luminosity. Most of the ULIRGs with Seyfert spectra however also show evidence for ongoing or recent star formation. Approximately $30 \%$ of the Seyferts are Sy1's, with a systematic increase compared to Sy2's with IR luminosity. Other notable findings included that optical reddening generally decreases with increasing distance from the nuclear regions and that the optically derived star formation rates are in most cases many times lower than those derived from mid/far-IR data. Followup spectroscopy in the near-IR (Veilleux. Sanders \& Kim, 1997, 1999b) refined and extended this picture, showing that, overall, around $25 \%$ of ULIRGs show evidence for an AGN and that this fraction increases with increasing IR luminosity (reaching $\sim 50 \%$ at $L_{i r}>10^{12.3} L_{\odot}$ ). Those ULIRGs with 'warm' IR colours are more likely to show broad lines in the near-IR than 'cool' ULIRGs, and there is no observed correlation between extinction in the NLR and the presence of broad lines in the near-IR, suggesting that the NLR and BLR do not lie along the same line of sight. An intriguing further result is that, of all the objects that show broad lines in the near-IR, all are Sy2's in the optical, with no LINERs or HII's. Furthermore, most $(\sim 70 \%)$ of the ULIRGs that show a Sy2 spectrum in the optical show broad lines in the near-IR.

Very recent spectroscopy of ULIRGs from the UV through to the near-IR has revealed some further important details. High spatial resolution UV and optical spectroscopy using the Space Telescope Imaging Spectrograph onboard HST of four 'warm' ULIRGs (Farrah et al., 2005) has shown that the 'knots' seen in optical imaging in many cases harbour very luminous starbursts and AGN, implying that these optically bright knots may also be the sites of the heavily obscured power sources behind the IR emission. The spectral properties of some of these knots also suggest further links between ULIRGs and (low ionization) broad absorption line (BAL) QSOs, and the forming cores of elliptical galaxies. The starbursts in these knots were all observed to be young, with ages of $4 \mathrm{Myr}-$ 20Myr, supersolar metallicities, and an IMF (Salpeter) slope of less than about 3.3. Near-IR spectroscopy with the Very Large Telescope (VLT) in Chile, and with Keck (Genzel et al., 2001; Tacconi et al., 2002) has shown that the host galaxy kinematics of ULIRGs resemble those of ellipticals with luminosities of $\sim L^{*}$ (but with a large scatter), and that the host galaxy properties of those ULIRGs that contain an AGN and those without are very similar. 
Finally, there is strengthening evidence that nuclear and galactic scale outflows may be common in ULIRGs (Heckman. Armus \& Milev, 1987; Wilman. Crawford \& Abraham, 1999), and high resolution spectroscopy has discovered galactic-scale outflows with high ejection efficiencies in many ULIRGs that are dominated by star formation (Rupke. Veilleux \& Sanders, 2002). Lipari et al. (2003) noted that relatively low velocity outflows are present in starburst dominated ULIRGs, but that higher velocity outflows are present in systems that show evidence for both a starburst and an AGN, though even in composite systems, the starburst is still probably the dominant mechanism behind galactic-scale outflows (Rupke. Veilleux \& Sanders, 2005).

\subsection{Mid-infrared spectroscopy}

An area where the Infrared Space Observatory, ISO (Kessler et al., 1996), excelled in the study of ULIRGs was in spectroscopy of nearby systems using SWS (de Graauw et al., 1996), LWS (Clegg et al., 1996), ISOCAM (Cesarsky et al., 1996) and ISOPHOT-S (Lemke et al., 1996), because for the first time highsensitivity and high-resolution spectroscopy could be obtained in a wavelength region with minimal effects of dust extinction, at least compared to the optical. Moreover the mid-infrared region holds several diagnostic lines which are very useful for characterization of the major power source for ULIRGs. The mid-infrared continuum shape can be a powerful diagnostic itself, betraying the existence of warm dust in the close vicinity of an AGN. We refer the reader to several previous reviews on ISO's legacy on star forming galaxies, AGN and ULIRGs (Genzel \& Cesarskv, 2000; Elbaz, 2005; Verma et al., 2005; Oliver \& Pozzi, 2005) for more details.

Genzel et al. (1998) demonstrated the power of SWS spectroscopy to separate AGN from starbursts by comparing the strength of the $7.7 \mu \mathrm{m}$ PAH equivalent width to high/low excitation line ratios, such as [OIV] $25.9 \mu \mathrm{m} /[\mathrm{NeII}] 12.8 \mu \mathrm{m}$; PAH molecules will be destroyed by high-intensity AGN radiation fields so low $\mathrm{PAH} /$ continuum ratios should correlate with strong $[\mathrm{OIV}] /[\mathrm{NeII}]$ ratios. They concluded that at leat half of their sample of 15 local ULIRGs have simultaneous starburst and AGN activity, but $70-80 \%$ of the sample is predominantly powered by star formation (a result mirrored by mid/far-IR SED fitting, Farrah et al. $(2003)$ ). The method requires high sensitivity to determine the fine structure line ratios, and the $\mathrm{PAH}$ emission can also be contaminated by strong silicate absorption at $9.7 \mu \mathrm{m}$. Moreover dense nuclear environments can hide AGN activity even at mid-IR wavelengths, as discovered by Clavel et al. (2000) in Seyfert 2 nuclei in which the the mid-infrared continuum from the AGN is sufficiently absorbed to allow extranuclear PAH emission to dominate the spectrum. Laurent et al. (2000) developed a diagnostic diagram based on the $6.2 \mu \mathrm{m}$ PAH feature relative to continuum strength versus mid-IR continuum colour, which could be used for systems lacking fine-structure line spectroscopy, and Peeters et al. (2004) extended this approach to include far-infrared continuum colours. Lutz et al. (1998); Rigopoulou et al. (1999) and Tran et al. (2001) applied these techniques to large ULIRG samples studied with ISOPHOT-S, concluding, in agreement 
with Genzel et al. (1998), that starbursts predominantly power these systems, though the presence of heavily obscured AGN cannot be ruled out.

Soifer et al. (2002) obtained low spectral resolution, but high angular resolution, Keck mid-IR spectra of five LIRGs - ULIRGs demonstrating that PAH emission, when present, generally is circumnuclear in origin, extended over scales of 100-500pc. The silicate optical depths in these sources can be as high as 15, suggesting that even mid-IR spectroscopy may not be probing the true nuclei in the most compact sources.

Mid-IR spectral classifications, however, generally agree with optical line classifications of AGN vs star formation power, although some ULIRGs with LINERlike optical spectra were interpreted as starburst dominated in the mid-IR, attributed to starburst wind-driven ionising shocks (Lutz. Veilleux \& Genzel, 1999; Sugai \& Malkan, 2000) instead of to low-level AGN. However, the LINER situation is complex as demonstrated by subsequent comparison of ISO-SWS fine structure line spectroscopy with Chandra X-ray imaging of a sample of LINERs. (Satvapal et al., 2004) has shown that LINERs are intermediate between starbursts and AGN in mid-infrared line excitation, and that most LINERs contain a compact hard X-ray source characteristic of an AGN. They also found some anti-correlations between mid-IR fine structure line diagnostics and hard X-ray AGN diagnostics: their highest excitation mid-IR spectrum source NGC 404 shows only weak soft X-ray emission while the low mid-IR excitation LINER NGC 6240 shows an extremely luminous binary X-ray AGN (Komossa et al., 2003). The most likely explanation for objects of this kind are extremely high optical depths even at mid-infrared and X-ray wavelengths, and potentially different lines-of-sight to the AGN core at different wavelengths (Risaliti et al., 2000), and/or unusual ratios of gas-to-dust optical depths.

Sturm et al. (2002) have developed diagnostic diagrams using [OIV] $25.9 \mu \mathrm{m}$, $[\mathrm{SiII}] 34 \mu \mathrm{m},[\mathrm{NeVI}] 7.65 \mu \mathrm{m}$ and $[\mathrm{NeII}] 12.8 \mu \mathrm{m}$ and other fine structure lines, coupled with Brackett $\beta$ at $2.63 \mu \mathrm{m}$, to separate high from low excitation systems and estimate the fractional contribution of star formation and AGN to the integrated light using mixture lines, in analogy to the well-known optical line diagnostic diagrams (Veilleux \& Osterbrock, 1987). Most ULIRGs were not detectable by ISO in these lines, though Arp 220 was shown to exhibit low excitation in these diagrams.

Spinoglio. Andreani \& Malkan (2002) proposed an analogous far-infrared approach using $[\mathrm{CII}] 158 \mu \mathrm{m},[\mathrm{OI}] 63 \mu \mathrm{m}$ and $[\mathrm{OII}] 88 \mu \mathrm{m}$, though again very few ULIRGs were detectable in these lines by ISO. Arp 220 has been observed extensively by ISO, as the nearest and brightest ULIRG. Arp 220's FIR spectrum is dominated by molecular absorption from species common to Galactic photodissociation regions (PDR): $\mathrm{OH}, \mathrm{H}_{2} \mathrm{O}, \mathrm{CH}, \mathrm{NH}, \mathrm{NH}_{3}$ (Fischer et al., 1999) and is very weak in the fine structure emission lines, with [OI] $63 \mu \mathrm{m}$ in absorption. The [CII] $158 \mu \mathrm{m}$ fine structure transition decreases with increasing IR luminosity (and more strongly with FIR colour $-S_{60 \mu m} / S_{100 \mu m}$ ) in infrared galaxies (Malhotra et al., 1997), and in ULIRGs is only $10 \%$ of that measured in less luminous starbursts (Fischer et al., 1999; Luhman et al., 2003). Since [CII] is the primary coolant in the ISM of normal galaxies it was expected to be strong in 
high-star formation systems, and its apparent lack is interpreted to indicate a stronger radiation field in luminous starbursts producing charged grains which result in a lower heating rate in PDRs (Malhotra et al., 2001). González et al. (2004) have modeled the FIR spectrum of Arp 220 over the spectral range 40$200 \mu \mathrm{m}$. Their model requires three components: the compact nuclei, modeled as a unit, with $T=10^{6} \mathrm{~K}$ which are optically-thick throughout the infrared, an extended region which is dominated by PDR emission at $T \sim 40-90 K$, and a halo which produces absorption from the low-lying levels of $\mathrm{OH}$ and $\mathrm{H}_{2} \mathrm{O}$ plus the CH. Given that the two nuclei show distinct mid-IR spectra (Soifer et al., 1999) it is unclear what effect modeling the nuclei as a single source may have. In this model the [CII] emission is produced in the extended (PDR) region with little [CII] emission from the nucleus, which has distinctly non-PDR conditions.

For the majority of ULIRGs for which spectroscopy is not available broadband colours have been used to characterize the broad spectral type and major infrared energy source, following the early IRAS schemes (Klaas et al., 2001; Spinoglio. Andreani \& Malkan, 2002; Farrah et al., 2003). ULIRGs are found to fall into two general classes by these methods: "cool" systems dominated by star formation, which show a relatively flat mid-infrared spectral shape and a steep rise towards longer wavelengths, and "warm" systems with a red powerlaw-like SED through the mid-infrared, usually assumed to be AGN-dominated, although compact HII regions can also show a warm mid-IR SED (Dopita et al., 2005). Warm ULIRGs tend to have Seyfert 1 like optical spectral features while cool ones have starburst or Seyfert 2 optical spectra. The two clases were found to be indistinguishable in the far-infrared (Klaas et al., 2001), suggesting that at these wavelengths the emission may not be dominated by an AGN in either class, however Peeters et al. (2004) find ULIRGs to have more prominent far-infrared emission than AGN or lower luminosity starbursts such as M82.

Several lines of evidence indicate that AGN become more bolometrically significant with increasing IR luminosity in ULIRGs. The incidence of both AGN optical line indicators and mid-infrared continuum and line AGN diagnostics were found to increase with increasing infrared luminosity (Shier. Rieke \& Rieke, 1996; Genzel et al., 1998; Lutz et al., 1998; Rigopoulou et al., 1999; Tran et al., 2001). However, these ISO HLIRG samples are biased towards previously known AGN so Spitzer confirmation of these result is important, using unbiased samples of infrared-selected HLIRGs.

Observations with the ISO satellite greatly expanded our understanding of the mid-infrared spectra of ULIRGs, however, many ULIRGs were beyond the reach of many of the diagnostic methods until the advent of Spitzer. Several extensive Spitzer programs are underway to adequately sample the local ULIRG population, the most comprehensive being a study of the mid-infrared spectra of a large number $(>100)$ of ULIRGs having $0.02<z<0.93$ with the Infrared Spectrograph (IRS), as part of the IRS guaranteed time program. These sources are chosen primarily from the IRAS 1-Jy (Kim \& Sanders 1998), 2-Jy (Strauss et al., 1992), and the FIRST/IRAS radio-far-IR sample of Stanford et al. (2000). In Figure 3 we show IRS spectra for three nearby ULIRGs (Mrk 1014, UGC 5101, and NGC 6240) whose spectra serve to highlight the range in properties 
seen throughout much of the sample, and the power of the IRS in wavelength coverage and sensitivity for studies of the nuclei and interstellar media in dusty galaxies (Armus et al., 2004; 2005).

Mrk $1014(z=0.1631)$ is a radio-quiet, infrared luminous QSO with broad optical emission lines and twin tidal tails indicative of a recent interaction (MacKenty \& Stockton 1984). UGC $5101(z=0.039)$ has a single, very red nucleus within a disturbed morphology suggestive of a recent interaction. Optically, UGC 5101 is classified as a LINER (Veilleux et al., 1995). It has a high brightness temperature $\left(T>10^{7} \mathrm{~K}\right)$ radio nucleus at $1.6 \mathrm{GHz}$ which is resolved with the VLBA (Lonsdale et al., 1995). ISO SWS and PHT-S spectroscopy (Genzel el al., 1998) indicate a powerful, circumnuclear starburst. Based upon its IRAS colours, UGC 5101 is classified as a cold, starburst-dominated, far-infrared source. However, XMM data indicate an obscured, but luminous, hard X-ray source with $L_{x}(2-10 \mathrm{keV}) \sim 5 \times 10^{42} \mathrm{erg} \mathrm{s}^{-1}$ and $L_{x}(2-10 \mathrm{keV}) / L_{I R} \sim 0.002$ suggestive of a buried AGN (Imanishi et al., 2003). NGC $6240(z=0.0245)$, is a doublenucleus, merging galaxy (Fosbury \& Wall 1979), with an $8-1000 \mu$ m luminosity of $\sim 7 \times 10^{11} L_{\odot}$. The optical nuclear spectrum of NGC 6240 is classified as a LINER (Armus, Heckman \& Miley 1987), and the extended optical nebula reveals the presence of a starburst-driven superwind (Heckman, Armus \& Miley 1987). X-ray observations with ASCA (Turner et al., 1998), Beppo-Sax (Vignati et al., 1999), Chandra (Komossa et al., 2003; Ptak et al., 2003), XMM-Newton (Netzer et al., 2005) provide clear evidence for the presence of one (or two) AGN behind significant columns of absorbing material $\left(N_{H}=1-2 \times 10^{24} \mathrm{~cm}^{-2}\right)$. Relatively strong [OIV] $25.89 \mu \mathrm{m}$ line emission in the ISO SWS spectrum of NGC 6240 led Lutz et al. (2003) to suggest that up to $50 \%$ of the infrared energy emitted by NGC 6240 could be powered by a buried AGN.

The spectra of Mrk 1014, UGC 5101, and NGC 6240 are strikingly different. Mrk 1014 has a steeply rising mid-infrared spectrum with weak emission features and little or no silicate absorption. The spectra of UGC 5101 and NGC 6240, on the other hand, are dominated by strong silicate absorption at $9.7 \mu \mathrm{m}$ and $18 \mu \mathrm{m}$, and PAH emission at 6.2, 7.7, 11.3, and $12.7 \mu \mathrm{m}$ (Armus et al. 2004, 2005). The extinctions toward the nuclei in UGC 5101 and NGC 6240, as estimated from the depths of the silicate absorption, are at least $\mathrm{A}_{V}=15-35$ and $\mathrm{A}_{V}=60 \mathrm{mag}$, respectively. UGC 5101 also shows strong absorption between $5-7.5 \mu \mathrm{m}$ from water ice and hydrocarbons. As suggested by Spoon et al. (2002), the water ice features may indicate the presence of shielded molecular clouds along the line of sight to the nucleus. NGC 6240 has little or no water ice absorption, but very strong emission lines from warm $(T \sim 300-400 \mathrm{~K}) \mathrm{H}_{2}$. The mass of this warm gas is estimated to be approximately $1.4 \times 10^{8} M_{\odot}-$ about $1 \%$ of the cold molecular gas mass derived from single-dish millimeter CO line measurements (Solomon et al. 1997), but up to $3-7 \%$ of the cold molecular gas within the central $1 \mathrm{kpc}$ measured by Tacconi et al. (1999).

The IRS high-resolution $(\mathrm{R}=650)$ spectra (not shown) provide important diagnostic measures of the dominant ionizing sources in the ULIRGs because it is possible to accurately measure unresolved atomic, fine-structure lines of $\mathrm{Ne}, \mathrm{O}, \mathrm{Si}$, and $\mathrm{S}$, covering a large range in ionization potential. The $[\mathrm{NeV}] 14.3$ 
/ [NeII] 12.8 and [OIV] 25.9 / [NeII] 12.8 line flux ratios in Mrk 1014 (0.9 and 1.7, respectively) suggests that nearly all the ionizing flux comes from the central AGN, although the obvious presence of PAH emission suggests some extranuclear star formation. In UGC 5101 and NGC 6240 weak $[\mathrm{NeV}]$ emission has been detected, with $14.3 \mu \mathrm{m}$ line fluxes of $5-6 \times 10^{-21} \mathrm{~W} \mathrm{~cm}{ }^{-2}$, indicating buried AGN. While the $[\mathrm{NeV}] /[\mathrm{NeII}]$ and the $[\mathrm{OIV}] /[\mathrm{NeII}]$ line flux ratios imply an AGN contribution of $<10 \%$ to the total luminosity in both sources (Armus et al. 2004, 2005), the large optical depth to the nuclei, as evidenced by the deep silicate absorption and X-ray columns, leaves open the possibility that the true contribution of the AGN to the bolometric power output in UGC 5101 and NGC 6240 may be much larger than revealed by the mid-IR emission lines. In NGC 6240, the extinction-corrected hard X-ray data are consistent with the buried AGN producing $50-100 \%$ of the luminosity. An inclined, dusty torus, patchy extinction, and/or a low covering factor for the $[\mathrm{NeV}]$-emitting clouds, could reconcile these apparently discrepant estimates - an explanation often evoked to explain observations of other type-2 AGN.

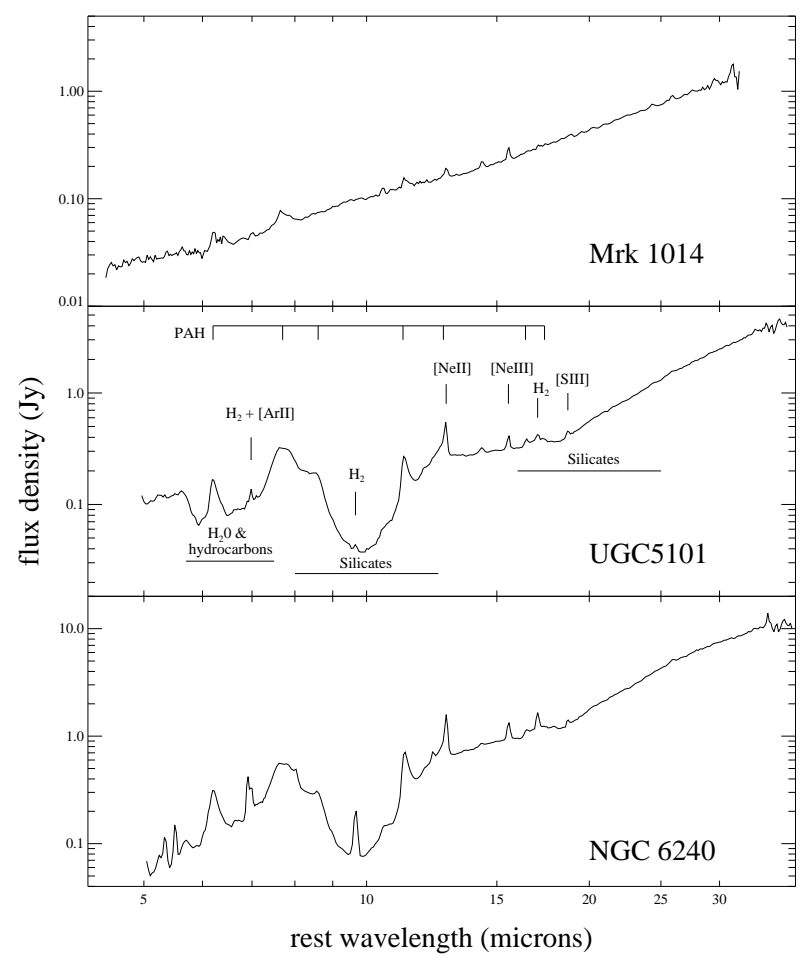

Fig. 3. IRS low-resolution spectra of Mrk 1014, UGC 5101 and NGC 6240. The positions of prominent emission features and absorption bands (the latter indicated by horizontal bars) are marked on the UGC 5101 spectrum. Figure courtesy of L. Armus and J. Houck. 
Spoon et al. (2004) observed the more distant ULIRG IRAS F00183-7111 at $\mathrm{z}=0.327$, detecting strong absorption from $\mathrm{CO}_{2} \& \mathrm{CO}$ gas, water ice, hydrocarbons and silicates, indicating high obscuration, a complex line of sight, and the presence of very warm, dense gas (720K). Direct signs of an obscured AGN are not found but Spoon et al. conclude that an obscured AGN probably accounts for most of the luminosity based on similarities to other systems with highly obscured AGN.

\subsection{Radio Continuum Studies: AGN vs Luminous Radio Supernovae}

The existence of a tight correlation between integrated far-infrared flux-density and radio continuum emission - the "Radio-FIR Relation" (Helou et al., 1985: Yun. Reddv. \& Condon, 2001) - over four orders of magnitude in IR luminosity, allows the use of high-resolution radio interferometric techniques to study the compact nuclei of luminous IR galaxies in one of the few spectral regions with relatively low optical depth. The Radio-FIR relation is not well understood, but is believed to be produced by nonthermal radiation from relativistic electrons in, or leaking out of, starburst-related supernova remnants. The seminal study of a complete sample of luminous IR galaxies by Condon et al. (1991) with the VLA at 1.49 and $8.44 \mathrm{GHz}$ demonstrated the extreme compactness of luminous IR nuclei and concluded that most of their 40 galaxies -6 of which are ULIRGs - are consistent with a starburst-related origin for the radio emission.

Lonsdale et al. (1993) used a global VLBI array to detect $18 \mathrm{~cm}$ high brightness temperature, $T_{b} \geq 10^{6} \mathrm{~K}$, emission cores from 17 of 31 luminous (log $\left.L_{F I R} \gtrsim 11.25\left(L_{\odot}\right)\right)$ infrared galaxies, consistent with such cores existing in all such LIRGs at $\sim 10 \%$ of the total $18 \mathrm{~cm}$ flux density. This result indicates either obscured radio-quiet AGN in most LIRGs and ULIRGs, possibly energetically dominant (Lonsdale et al., 1995), or clumps of starburst-related luminous radio supernovae (LRSN) and remnants (Smith et al., 1998). Similar studies in the South (Norris et al., 1990; Kewlev et al., 2000) detected compact nuclear radio cores with lower frequency, but their results are consistent, allowing for differences in sample selection and sensitivity.

Subsequent VLBI imaging dramatically revealed a cluster of luminous RSN in the nearest ULIRG, Arp 220 (Smith et al., 1998) - clear evidence for a high star formation rate, estimated at that time as $\sim 100 M_{\odot}, \mathrm{yr}^{-1}$ by Smith. Lonsdale \& Lonsdale (1998), which would be enough to power the infrared luminosity by star formation without resorting to an obscured AGN. Momiian et al. (2003); Neff. Ulvestad \& Teng (2004); Bondi et al. (2005) have reported possible "supernova factories" in IRAS 17208-0014, Mrk 299 and Mrk 273. respectively. The original dozen LRSN in Arp 220 have been monitored with VLBI (Rovilos et al., 2005) and more sensitive VLBI imaging studies (Lonsdale et al., 2006a) have revealed a number of new and fainter LRSN. These Luminous RSN were originally modeled after RSN1986J in NGC 891 (Weiler et al., 1990), which exhibited a maximum radio power, $\log P_{1.5 \mathrm{GHz}}^{1986 \mathrm{~J}}=$ 21.15. The LRSN in Arp 220, however, exhibit much slower decay in their radio light curves (Rovilos et al., 2005) than RSN 1986J; longer RSN lifetimes reduce 
the inferred star formation rate, therefore it is not clear from the VLBI imaging whether a starburst is the chief power source for Arp 220.

On the other hand (Lonsdale et al., 2003a) demonstrated that the radio emission in UGC 5101, Mrk 231 and NGC 7469 is AGN dominated. Mrk 231, in some senses the classical "infrared quasar", has been studied with the VLBI by Ulvestad et al. (1999). Their images (see also Lonsdale et al. 2003a) show a triple structure, with a core and two lobes which classify it as a Compact Symmetric Object (CSO). It has been suggested that CSOs are young, $\tau<<10^{6} \mathrm{yr}$, with the hot spots representing the working surface of a relativistic jet upon the ambient medium (Readhead et al., 1996). If the southern (primary) lobe/hot-spot in Mrk 231 is confined by ram pressure, (Lonsdale et al., 2003a) estimate a lobe advance speed, $v_{a} \sim 10^{-4} c$ and an age for the jet/compact source, $\tau<10^{6} \mathrm{yr}$. Despite the clear evidence for AGN domination of the radio structure in these systems, the radio power is small compared to the infrared/bolometric emission, which may still therefore be starburst dominated. In Mrk 231, for example, several studies (Carilli et al., 1998; Lonsdale et al., 2003a; Farrah et al., 2003) suggest that more than half of the total luminosity comes from a circumnuclear starburst in a molecular ring rather than from the AGN.

\subsection{Molecular gas: CO \& HCN Observations}

The interpretation of Luminous Infrared Galaxies as starburst systems was strengthened soon after their identification with IRAS by studies of neutral hydrogen (Mirabel \& Sanders, 1988) molecular gas, principally CO (Sanders et al., 1986, 1991) but more recently HCN (Gao \& Solomon, 2004) and OH maser emission (Baan et al., 1982; Baan, 1989). These early studies demonstrated that ULIRGs as a class exhibit compact nuclear reservoirs of high-density gas, and, with mass estimates of order $10^{9}-10^{10} M_{\odot}$ in $\mathrm{HI}$ and in $\mathrm{H}_{2}$, are consistent with the interpretation that star formation accounts for a substantial fraction of the FIR luminosity in Luminous IR Galaxies.

Millimeter-wave interferometer measurements of CO emission (e.g. Scoville et al. 1991; Solomon et al. 1997; Downes \& Solomon 1998; Bryant \& Scoville 1999) have demonstrated that nearly half of the CO mass in Luminous IR Galaxies is contained within the central regions, $r<0.5-1 \mathrm{kpc}$, with as much as $10^{10} M_{\odot}$ of molecular gas distributed in nuclear disks of radius a few hundred pc, but thicknesses of one-tenth the radius and densities over $10^{4} \mathrm{~cm}^{-3}$ (Brvant \& Scoville, 1999). The mean molecular surface densities in these structures may exceed $10^{4} M_{\odot} \mathrm{pc}^{-2}$ with the molecular gas providing a large fraction of the dynamical mass. The picture of molecular gas in ULIRGs is very different from that in the Galaxy, with much higher surface densities and inferred optical depths $\left(A_{v} \gtrsim 10^{2}-10^{3}\right)$. Early $\mathrm{CO}$ studies assumed a Galactic conversion ratio between CO Luminosity and $\mathrm{H}_{2}$ mass, $M_{H_{2}} / L_{C}^{\prime} O=4.6 M_{\odot}\left(\mathrm{K} \mathrm{km} \mathrm{s}^{-1} \mathrm{pc}^{-2}\right)$, however, recent analyses suggest that this may overestimate the molecular mass, either owing to high-brightness temperatures in the CO emission (e.g. Mrk 231; Bryant \& Scoville 1999) or because the line width/velocity dispersion reflects 
the total dynamical mass in the central nuclear region, rather than that within virialized molecular clouds (Solomon et al., 1997).

Again, Arp 220 provides a convenient laboratory to study molecular gas, if not always in a typical ULIRG environment. Sakamoto et al. (1999) have studied the Arp 220 nuclei in the $\mathrm{CO}(2-1)$ transition and accompanying $1 \mathrm{~mm}$ continuum. Steep velocity gradients are found in the $\mathrm{CO}$ associated with each of the two nuclei, which are misaligned with each other and with the outer CO disk. Sakamoto et al. interpret these as molecular disks associated with the merging nuclei, counter-rotating with respect to each other and with respect to the outer disk. The dynamical masses inferred from $\mathrm{CO}$ kinematics are of the order of $2 \times 10^{9} M_{\odot}$. The central molecular gas is inferred to have high filling factor, more like a uniform disk than individual clouds, which will have an inward accretion rate of approximately $100 M_{\odot} \mathrm{yr}^{-1}$ (Scoville. Yun \& Brvant, 1997) which is similar to the star formation rate of Arp 220 inferred from the FIR Luminosity (Smith. Lonsdale \& Lonsdale, 1998; Farrah et al., 2003).

Early studies placed considerable emphasis on the ratio of FIR Luminosity to CO Luminosity - often called the Star Formation Efficiency - demonstrating that the ratio of FIR/CO increases at higher $L_{F I R}$. This is interpreted as either an increase in Star-Formation Rate per unit molecular gas mass, i.e. more efficient star formation, or as possible evidence for an alternative, AGN, contribution to the FIR luminosity (Sanders \& Mirabel 1996). More recently, studies of $\mathrm{HCN}$, which, owing to the higher dipole-moment of the HCN molecule is a tracer of warmer $\left(T_{k i n} \sim 60-90 \mathrm{~K}\right)$, higher-density $\left(n_{H_{2}} \sim 10^{5}-10^{7} \mathrm{~cm}^{-3}\right)$ gas, shows a much higher ratio of $\mathrm{HCN} / \mathrm{CO}$ luminosity in Luminous IR Galaxies than in quiescent spirals like the Milky Way (Solomon. Downes \& Radford, 1992). Furthermore the FIR/HCN luminosity (HCN Star Formation Efficiency, as above) shows an approximately linear relationship in Luminous IR Galaxies with $L_{\text {fir }}$ from $10^{10}-10^{13} L_{\odot}$ indicating the presence of an abundant warm, high-density molecular environment within ULIRGs (Gao \& Solomon, 2004). ULIRGs thus

have a much greater dense, warm medium like that of the star-forming cores of Galactic molecular clouds - perhaps the central regions of the molecular disks, although little spatial information is available about the HCN distribution in ULIRGs. Since there is little doubt that the lower luminosity systems amongst this sample are dominated by star formation, the continuity of this relation up to luminosities characteristic of HLIRGs is one of the most compelling pieces of evidence, albeit statistical, that most ULIRGs are dominated by starburst power rather than AGN power.

\subsection{Maser Emission}

Luminous IR Galaxies have been known to be strong emitters of $\mathrm{OH}$ maser emission since the discovery of $\mathrm{OH} 1667 \mathrm{MHz}$ emission in Arp 220 by Baan et al. (1982), who dubbed it a "megamaser", having an OH luminosity roughly $10^{6}$ times that of masers in the Galaxy. $\mathrm{H}_{2} \mathrm{O}$ maser emission, which has been used to map torus/disklike structures around compact supermassive AGN nuclei such as 
NGC 4258 (Mivoshi et al., 1996) is not generally detected in classical Luminous IR Galaxies (see Lo 2005 for a review).

$\mathrm{OH}$ megamasers are found preferentially in the most luminous IR galaxies (Baan, 1989), and a rough dependence of the $\mathrm{OH}$ luminosity on the square of the FIR luminosity was originally found, suggesting that the FIR emission provided the pumping mechanism to provide the necessary population inversion (e.g. Henkel \& Wilson 1990) within a foreground molecular screen amplifying the diffuse radio continuum, which is in turn correlated with the FIR emission. The $\mathrm{OH}$ gas may trace very high density regions $\left(\mathrm{n}_{\mathrm{H}_{2}}=10^{5-7} \mathrm{~cm}^{-3}\right)$, although an apparently separate component of the $\mathrm{OH}$ megamasers is associated with a much lower-density high-velocity outflow (Baan. Haschick \& Henkel, 1989).

The initial interpretation of the maser phenomenon of a foreground, lowgain molecular screen amplifying the diffuse radio continuum in Luminous IR Galaxies, however, has been questioned by the VLBI observations of Arp 220 (Diamond et al., 1989; Lonsdale et al., 1994, 1998; Rovilos et al., 2003) demonstrating that over two-thirds of the masing gas in Arp 220 is compact, produced in structures a few $\mathrm{pc}^{3}$ in volume with amplification ratios of order $10^{3}$ or higher. These compact masers have complex spatial and velocity structure arising in clouds generally within the Arp 220 nuclei, but not coincident with nuclear radio continuum (or any other detectable radio continuum). The southern component within the western nucleus (the one with the majority of LRSN) shows a velocity gradient in excess of $18,000 \mathrm{~km} \mathrm{~s}^{-1}$ (Rovilos et al., 2003), which, interpreted as rotation, implies a mass of order $2 \times 10^{7} M_{\odot}$.

Similar compact maser emission has been detected with Global VLBI in IIIZw35 and IRAS17208-0014 (Diamond et al., 1999), and in 12032+1707 (Pihlstrom et al., 2005). On the other hand Mrk 231 (Lonsdale et al., 2003a; Klöckner et al., 2003), Mrk 273 (Klöckner \& Baan, 2004) and 14070+0525 (Pihlstrom et al., 2005) show more extended emission in a circumnuclear disk with conditions more like the classical maser model. IIIZw35 also shows diffuse ring of maser emission with radius $\sim 20 \mathrm{pc}$ and central mass $\sim 7 \times 10^{6} M_{\odot}$ (Pihlstrom et al., 2001). In this case the compact emission occurs at the tangent points of the ring, and the maser structure has been modeled by multiple high density clouds within the diffuse ring. Such a structure does not, however, appear to be able to explain the complex, compact maser emission in Arp 220.

In the case of Mrk 231, the $\mathrm{OH}$ emission appears to be the central portion of a circumnuclear HI disk detected in absorption by Carilli et al. (1998), though it is misaligned with the central CO distribution (Klöckner \& Baan, 2004). Lonsdale et al. (2003a) speculate that the ignition of Mrk 231's Seyfert nucleus may have disrupted compact maser emission in the nucleus itself.

An extensive survey for $\mathrm{OH}$ maser emission in over 300 IRAS galaxies from the PSCz Survey (Saunders et al., 1990) with $z>0.1$ has been carried out with the upgraded Arecibo Telescope by Darling \& Giovanelli (2002 and references therein). This survey has expanded the sample of known megamasers in luminous infrared galaxies to over 100 with detections in nearly $20 \%$ of the galaxies surveyed. Their analysis suggests few correlations between $\mathrm{OH}$ and FIR properties. A re-analysis of the $L_{O H}-L_{F I R}$ relationship in their data sug- 
gests a much flatter relation $L_{O H} \propto L_{F I R}^{1.2}$ consistent with other recent analyses Kandalian, 1996) which finds a slope of approximately 1.4, suggesting a mixture of extended, unsaturated emission plus compact, saturated clouds. One source in the Darling \& Giovanelli (2002) sample, IRAS 21272+2514, shows apparent variability which the authors interpret as due to interstellar scintillation. This interpretation requires $\sim 30-60 \%$ of the $\mathrm{OH}$ maser emission to originate in saturated clouds of dimension smaller than $\sim 2 \mathrm{pc}$.

A satisfactory model for the complexities of $\mathrm{OH}$ masers in ULIRGs has yet to be proposed. Almost certainly the diffuse emission follows the classical model with largely unsaturated $\mathrm{OH}$ maser clouds, pumped by the strong FIR radiation field, amplifying the nuclear $1.6 \mathrm{GHz}$ radio continuum. The compact, pc-scale structures in Arp 220 and other galaxies, coupled with the lack of detectable associated $1.6 \mathrm{GHz}$ continuum emission, requires a substantial saturated maser component. These structures subtend solid-angles too small to intercept sufficient FIR photons to excite the $\mathrm{OH}$ molecules, suggesting that collisional excitation must be important (Lonsdale et al., 1998). A tentative correlation between $\mathrm{OH}$ line-width and X-ray luminosity (Kandalvan, 2003) may indicate that X-ray heating of the molecular gas plays a role in collisional excitation. The possible roles of shocks and/or AGN activity remain to be explored.

\subsection{X-Ray Emission}

The importance of X-rays has been recognized not only because of the diagnostic ability of the X-ray to discriminate between AGN and Starburst emission (Rieke, 1988), but also because models of the X-ray background (XRB) require substantial populations of highly-obscured AGN at redshifts, $z \sim 0.5-1.5$, to reproduce the observed XRB spectrum (Worsley et al. 2005). Rieke's early analysis from HEAO A-1 demonstrated that ULIRGs are underluminous in the $2-10 \mathrm{keV}$ band compared to classical Seyfert galaxies or QSOs. It has only been recently with the Chandra and XMM-Newton X-ray observatories that the implications of this early discovery could be investigated in significant ULIRG samples with sufficient resolution and signal-to-noise to provide reliable diagnostics of the nature of the nuclear X-ray sources.

Further evidence of the weakness of X-ray emission in ULIRGs comes from a ROSAT survey (Boller et al., 1998) of 323 ULIRGs which detected fewer than $10 \%$. Probably the most significant starburst-related discovery of the ROSAT soft X-ray satellite is the detection of extended thermal outflows, first in nearer, lower-luminosity starbursts, and then in Arp 220, dubbed "superwinds" (Heckman et al., 1996). Similar thermal components appear to be common in ULIRGs and they are described as "ubiquitous" in starbursts with Star Formation Rates $>10^{-1} M_{\odot} \mathrm{yr}^{-1} \mathrm{kpc}^{-2}$ in Heckman's (2001) review; see also the recent review by Veilleux. Cecil. \& Bland-Hawthorn (2005). The superwinds are believed to be driven by supernova supplied kinetic energy with outflow rates comparable to the star-formation rates in these galaxies. Heckman speculates that these superwinds may be the principal "polluters" of metals and dust into 
the IGM. Further Chandra studies (McDowell et al., 2003) of the Arp 220 superwind reveal extended, faint, edge-brightened, soft X-ray lobes outside the optical galaxy out to a distance of 10-15 kpc. Bright plumes inside the optical isophotes coincide with the optical line emission (Colina et al., 2004) and extend $11 \mathrm{kpc}$ from end to end across the nucleus. The data for the plumes cannot be fitted by a single-temperature plasma and display a range of temperatures from 0.2 to $1 \mathrm{keV}$. There is a close morphological correspondence between the $\mathrm{H} \alpha$ and soft $\mathrm{X}$-ray emission on all spatial scales.

There have been three recent ULIRG X-Ray surveys with XMM-Newton (Franceschini et al., 2003a) which observed 10 ULIRGs, and with Chandra (Ptak et al., 2003; Teng et al., 2005) which observed 8 and 14 respectively. All of the ULIRGs surveyed were detected, with X-ray luminosities typically $L_{2-10 \mathrm{keV}}<10^{42}-10^{43} \mathrm{erg} \mathrm{s}^{-1}$. These luminosities represent $<1 \%$ of the infrared luminosities in these systems, confirming that ULIRGs are much less luminous in the X-ray than classical AGN. Furthermore, the soft X-ray emission from all systems is dominated by extended, thermal emission with $k T \sim 0.7 \mathrm{keV}$, and is uncorrelated with IR luminosity. In at least two XMM-Newton systems the emission is extended on scales of $10 \mathrm{~s}$ of kpc, suggesting a superwind origin. In at least 5 galaxies a hard X-ray $(2-10 \mathrm{keV})$ component and/or the presence of $6.4 \mathrm{keV}$ Fe K-line emission suggests the presence of an AGN which is not energetically dominant (Franceschini et al., 2003a). Similarly, in the nearer Chandra sample of Ptak et al. (2003) all galaxies exhibit hard components which are interpreted as AGN sources. 5 galaxies in the Chandra samples which are classed as AGN (including IRAS 05189-2524, Mrk 231, Mrk 273) show order of magnitude greater X-ray luminosities than the Starburst ULIRGs (UGC 5101, IRAS 17208-0014, IRAS 20551-4250, IRAS 23128-5919) with NGC 6240 being an intermediate case ${ }^{6}$. As already noted UGC 5101 and Mrk231 are believed to harbor obscured AGN based on their Spitzer spectra. Mrk 273, NGC 6240 and UGC 5101 exhibit Fe K-line emission, but sufficiently weak that the authors argue against X-rays reflected from a Compton-thick $\left(N_{H}>10^{24} \mathrm{~cm}^{-2}\right)$ absorber as the origin of the observed X-ray emission. Teng et al. (2005) use hardness ratios to estimate the X-Ray spectral properties of their fainter galaxies. The photon indices for the combined Chandra samples peak in the range $1<\Gamma<1.5$, with a tendency for Seyfert ULIRGs to have steeper spectra, $\Gamma>2$. Although the $\mathrm{X}$-ray properties of this fainter sample are consistent with a Starburst origin, the presence of Compton-thick AGN cannot be ruled out and may be expected in many cases given the results discussed above.

Although the situation is complicated, certainly some ULIRGs must harbor luminous X-ray AGN, though perhaps behind large absorbing columns and thus only visible in hard X-rays. In Mrk 231 BeppoSAX revealed a highly absorbed $\left(N_{H} \sim 2 \times 10^{24} \mathrm{~cm}^{-2}\right)$ power-law component (Braito et al., 2004) and analysis of XMM-Newton data indicates that below $10 \mathrm{keV}$ only scattered or reflected X-rays escape. In a 40ks observation of of Mrk 231, Gallagher et al. (2002) find

\footnotetext{
${ }^{6}$ Mrk 231, IRAS 17208-0014 and IRAS 23128-5919 are included in both the Ptak et al. (2003) and Franceschini et al. (2003a) samples
} 
the majority of the X-ray luminosity is emitted from an unresolved nuclear point source with a very hard spectrum, the majority of the flux emitted above $2 \mathrm{keV}$. The source is also variable on a timescale of a few hours. They argue against a Compton-thick reflection model of Mrk 231 (Malonev \& Revnolds, 2000), proposing a Compton-thick absorber which allows scattered light from multiple lines of sight to be detected. Highly absorbed AGN power-law hard Xray sources are also reported in Mrk 273 (Xia et al., 2002: Balestra et al., 2005), NGC 6240 (Iwasawa \& Comastri, 1998; Vignati et al., 1999; Netzer et al., 2005), and Mrk 1014 (Boller et al., 2002) although the starbursts in these galaxies dominate in softer X-rays, and possibly also bolometrically.

Again the nearby system, Arp 220, provides more details, as well as unanswered questions. BeppoSAX observations (Iwasawa et al., 2001) placed severe constraints on an energetically significant AGN in Arp 220, requiring an absorbing column, $N_{H}>10^{25} \mathrm{~cm}^{-2}$ to hide an X-ray AGN. They suggested X-ray binaries as the source of Arp 220's hard X-rays. Clements et al. (2002) detected several sources near Arp 220's nucleus, including a mildly absorbed point source with a hard spectrum coincident with the western radio nucleus, plus a fainter source which may coincide with the eastern nucleus. A classical X-ray AGN cannot be ruled out, but again, columns greater that $5 \times 10^{24} \mathrm{~cm}^{-2}$ would be required to hide it. Iwasawa et al. (2005) have reported the detection of Fe K emission in the XMM-Newton spectrum of Arp 220. A supernova shocked bubble as suggested by the VLBI observations of radio supernovae could provide an explanation. However, the apparent lack of emission from X-ray binaries is

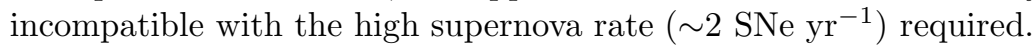

\section{The emerging picture of ULIRGs: Local Universe}

Taken as a whole, the observations of local Luminous Infrared Galaxies suggest that, at lower luminosities at least, they are dominated by starburst emission. The continuity of the $L_{H C N}$ vs $L_{F I R}$ relation (Gao \& Solomon, 2004) provides circumstantial evidence that most, if not all, Luminous IR Galaxies are starburst powered. Their sample, however, as well as many others quoted above, suffers from the low space density of ULIRGs, with only 6 classical ULIRGs $\left(L_{I R}>10^{12} L_{\odot}\right)$ and only two with $\left.L_{I R}>10^{12.2} L_{\odot}\right)$. The LRSN discovered in compact ULIRGs would seem to indicate starburst dominance in these systems, however we do not understand enough about these LRSN to establish a starformation rate or a starburst bolometric luminosity, leaving open the possibility of significant if not dominant AGN power even in the best studied source Arp 220. ULIRGs are generally under-luminous in the X-rays compared to classical AGN, requiring sensitive, high energy, observations to detect them. Evidence for very high X-ray obscuring columns is mounting, which could explain the low observed flux levels, and estimates of the importance of the X-ray-detected, obscuration-corrected AGN power to ULIRG energetics range from minor to dominant. Spectroscopy from the optical through mid-infrared suggests that the incidence of AGN and their strength relative to the ubiquitous starbursts in- 
creases with FIR Luminosity and that AGN may dominate for $L_{I R}>10^{12.5} L_{\odot}$, but there are very few such ULIRGs in most local samples. Mid-infrared high excitation diagnostic lines are a very promising line of investigation, however very deep silicate and ice absorptions are also detected in some ULIRGs, so even these mid-IR diagnostics may severely under-estimate AGN energetic contributions due to extinction.

And so the debate continues, and the very fact that it continues two decades after IRAS highlights that most ULIRGs almost certainly contain both a starburst and a monster, and that the key question really concerns the connection between the two, be it evolutionary (one evolves into the other), causal (one triggers the other somehow) or coincidental (another influence triggers both). We cannot address the wealth of literature on this topic here, involving as it does evolutionary schemes of all kinds and their implications, and extensive comparative AGN and starburst population studies and demographics, and so we outline here only a few key points. One popular scenario is an evolutionary sequence in which major gas rich galaxy mergers first result in a massive cool starburstdominated ULIRG, followed by a warm ULIRG as a QSO turns on inside the dust cocoon and heats the surrounding dust, and then finally the QSO emerges in an optically bright phase when it blows away the surrounding dust cocoon, and the resulting stellar system resembles a spheroid (Sanders et al. 1988a; Kormendy and Sanders 1992; Joseph 1999; Fabian 1999; Lipari et al. 2003). Another important scenario is unification-by-orientation of AGN, in which a broad-line (type I) active nucleus is highly obscured by a dusty molecular torus (or other non-symmetrical geometry) when viewed off-axis, so that an optically bright QSO viewed off-axis would appear as a ULIRG. Both of these scenarios predict close relationships between starbursts and AGN.

The evolutionary scenarios have recently received a boost from a sequence of papers reporting high resolution hydrodynamic simulations of gas rich major mergers (Di Matteo et al., 2005; Springel et al., 2005; Hopkins et al., 2005a,b,c,d), motivated by linking the growth of spheroid masses and supermassive black hole (SMBH) masses in order to explain the the observed correlations between SMBH mass and bulge mass (Magorrian et al. 1998) or velocity dispersion (Ferrarese and Merritt 2000, Gebhardt et al. 2000) of local spheroids. Accretion rates are predicted to be highest at late merger stages, when the SMBH grows exponentially, followed by the most luminous optically-visible QSO phase when the active QSO essentially explosively drives out all remaining material in the system (Hopkins et al. 2005a). The period of high obscuration during the high accretion rate phase would correspond to an obscured QSO, ie an AGN-powered ULIRG. Starburst events occur earlier in the lifetime of the merger when gas is still plentiful, and a starburst-ULIRG phase could occur when the gas is centrally concentrated into a dense compact region at relatively late stages.

Hopkins et al. (2005e) have made approximate predictions of ULIRG space densities based on these simulations, estimating at $\mathrm{z}=0.15,3 \times 10^{-7}$ and $9 \times 10^{-8}$ $\mathrm{Mpc}^{-3}$ at infrared luminosities of 1.6 and $2.5 \times 10^{12} L_{\odot}$, respectively, which is in good agreement with the 1Jy survey of Kim and Sanders (1998). Hopkins et al. also predict a decrease by a factor of 1.5 to the lower redshift, $\mathrm{z}=0.04$, 
which also agrees well with the Kim and Sanders work, and an increase to z 13 of $\Phi\left(L>10^{11} L_{\odot}\right)$ of $1-3 \times 10^{-5} \mathrm{Mpc}^{-3}$, which they compare to the submm number counts of Barger et al. (2005). Chapman et al. (2005) have derived the first estimate of ULIRG luminosity functions in this redshift range, though sensitive only to systems above about $10^{12.3} L_{\odot}$. They find space densities $>6$ $\times 10^{-6} \mathrm{Mpc}^{-3}$ in their luminosity range, which could be significantly higher than the Hopkins et al. predictions, depending on the unmeasured break of the IR ULIRG luminosity function at these redshifts. Deep Spitzer surveys should help extend the ULIRG luminosity function to lower luminosities in this redshift range.

These ULIRG-QSO evolutionary sequences are appealing and seem likely to be correct for at least a fraction of the ULIRG population. Many predictions remain to be verified, however, and alternative scenarios are also popular. A key test is the host morphology of ULIRGs and optically-bright QSOs. As we have described in Section 3, not all ULIRGs are found at late stages of a merger, although most are indeed found to be associated with the merger process. Farrah et al. (2001) have proposed an alternative merger-evolution scheme in which the stage at which a ULIRG occurs depends chiefly on the morphological type of the two interacting systems. Deep HST imaging of QSO hosts is now revealing that significant numbers also appear to inhabit disturbed systems (Lim \& Ho, 1999; Percival et al., 2001; Sánchez et al., 2004), supporting an evolutionary connection between starbursts and QSOs, although some QSOs appear to occur in spiral hosts with very little evidence of recent disturbance. This latter result has been taken to indicate that they could not have been triggered by a recent major merger, however recent simulations have demonstrated that major gas-rich mergers can result in disk systems under certain circumstances (Springel \& Hernquist, 2005; Robertson et al., 2005)

Genzel et al. (2001) and Tacconi et al. (2002) observed NIR structural properties and stellar dynamics of $18 \mathrm{z}<0.18$ ULIRGs, showing that ULIRG hosts lie on the fundamental plane of elliptical galaxies and thus are very likely to evolve into ellipticals, but that they have significantly lower mass hosts than a luminosity-matched sample of radio-loud and radio-quiet QSOs from Dunlop et al. (2003). A difficulty with this approach is that both starbursts and QSOs are expected to have rapidly varying luminosity during the merger sequence, so it is not clear that samples should be luminosity matched to compare their host masses. These authors also reviewed the cluster environment of local ULIRGs, QSOs (from the study of McLure \& Dunlop 2002) and ellipticals, finding that while the ellipticals and quasars are found in all environments, none of the 117 ULIRGs that were investigated are located in an environment richer than a small group, which provides some statistical evidence that at least those quasars found locally in rich environments may not have evolved via a ULIRG phase.

Haas et al. (2003) searched for a red transition population between obscured and unobscured QSO populations amongst Palomar-Green (PG) QSOs. Optically-selected QSOs as a class have a very high incidence rate of luminous FIR and submm emission, consistent with most of them qualifying as ULIRGs by IR luminosity level (Haas et al., 2003; Polletta et al., 2000; Hatziminaoglou 
et al., 2005). Haas et al. suggested a sequence of near-, mid- and far-infrared SEDs for their PG QSO sample which could plausibly represent the transition from a young dust-obscured QSO through the stages of re-distribution of the dust and settling of the dust into a torus, and which is less well explained by simple orientation effects alone. Late evolution-stage systems may be expected to have low fuel supplies, perhaps as seen some moderate redshift HLIRGs with low CO masses and $L_{I R} / M_{\text {gas }}$ ratios or which lack cool dust components (eg. Yun \& Scoville 1998; Verma et al., 2002). Another suggested young QSO transitory class are the broad absorption line (BAL) QSOs, especially those with strong FeII and weak [OIII] emission, which are preferentially found to be infrared luminous and which could therefore be young dusty QSOs (Voit et al., 1993; Lipari, 1994; Egami et al., 1996; Canalizo \& Stockton 2001, Lipari et al., 2003). FeLoBAL QSOs could alternatively be older QSOs viewed preferentially along the radial surface of the torus (Elvis 2000).

Another class of potentially young broad-line QSOs are the red 2MASS QSOs, selected to have J-K $>2$ and expected to be significantly dust-obscured (Cutri et al. 2002). These objects tend to be type-1 AGN with moderate luminosities and $\mathrm{z}<0.8$, the relatively low luminosities and redshifts being due to a selection bias due to the near-infrared k-correction. Leipski et al. (2005) extended this red 2MASS QSO search using ISO $6.7 \mu \mathrm{m}$ data, and found a surface density of 1.5 times that of SDSS QSOs to the same optical magnitude depths, and also that the 2MASS-ISO QSOs are significantly redder than SDSS QSOs, and that SDSS QSO colour selection criteria would have missed about $1 / 3$ of these red QSOs.

Most detailed imaging and morphological studies so far have focused on relatively low redshift systems. At higher redshifts we might expect different trigger-

ing mechanisms under different environmental circumstances, which we discuss further in Section 5.

\section{ULIRGs at high redshift}

\subsection{The cosmic infrared background}

The existence of a significant population of IR-luminous galaxies at high redshift, though hinted at in followup of IRAS, was thrown to the fore of astronomical debate by results from the Cosmic Background Explorer (COBE) satellite. Launched on November 18, 1989, COBE was responsible for two of the most significant astronomical results in the 20th century. The first was measuring the spectral shape and level of anisotropy in the Cosmic Microwave Background (CMB, peaking at $\sim 2000 \mu \mathrm{m})$, providing overwhelming evidence for the Hot Big Bang model and giving a glimpse at the early structures that would eventually evolve into todays galaxies and clusters. The second result, more relevant for this review, was the discovery of a Cos-

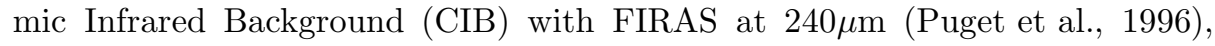
and at $140 \mu \mathrm{m}$ and $240 \mu \mathrm{m}$ with DIRBE (Schlegel. Finkbeiner \& Davis, 1998; 
Hauser et al., 1998; Fixsen et al., 1998) (later detections with DIRBE of the CIB at $2.4 \mu \mathrm{m}$ and $3.5 \mu \mathrm{m}$ have been published, see Hauser \& Dwek, 2001; and Kashlinsky, 2005 for reviews). Such an infrared background had been predicted many years previously (Partridge \& Peebles, 1967), but had proven fiendishly difficult to detect, remaining invisible both to rocket-borne IR observatories (Kawada et al., 1994), and to IRAS (Rowan-Robinson et al., 1990; Oliver. Rowan-Robinson \& Saunders, 1992). COBE was the first observatory with both the right instrumentation and sufficient sensitivity to detect the CIB.

The cosmological implications from the discovery of the CIB were profound (Dwek et al., 1998). The total background detected by COBE between 140 and $5000 \mu \mathrm{m}$ is $\sim 16 \mathrm{nW} \mathrm{m}^{-2} \mathrm{sr}^{-1}$, or $20 \%-50 \%$ of the total background light expected from energy release by nucleosynthesis over the entire history of the Universe, implying that 5\%-15\% of all baryons are or have been parts of stars. And while the CIB itself amounts to less than about $2 \%$ of the CMB, the intensity of the CIB is still surprisingly high, comparable to, or exceeding, the integrated optical light from the galaxies in the Hubble Deep Field (Hauser et al., 1998).

When compared to the cosmic history of star formation derived from optical and UV surveys (Madau et al., 1996) a serious discrepancy became apparent; the CIB detected by COBE requires at least a factor of two more star formation than was apparent in optical and UV surveys, meaning that the integrated star formation rate at $z \sim 1.5$ must be higher than that implied from UV/optical observations by a comparable factor, and that this star formation must be largely surrounded by dust. COBE however could provide little further constraint on the form of the star formation history implied by the CIB; in principle there could be large numbers of galaxies at high redshift that are faint in the IR, or a few extremely IR-luminous sources ${ }^{7}$. Further progress required that the CIB be resolved into its constituent sources.

\subsection{Resolving the CIB: LIRGs and ULIRGs at $0<z<1.5$}

The first major steps in resolving the CIB came from extragalactic surveys carried out by ISO at $7 \mu \mathrm{m}$ and $15 \mu \mathrm{m}$ with ISOCAM, and at $90 \mu \mathrm{m}$ and $170 \mu \mathrm{m}$ with ISOPHOT, most notably observations of the HDF (Oliver et al., 1997; Rowan-Robinson et al., 1997), the European Large Area ISO Survey (ELAIS; Oliver et al., 2000; Rowan-Robinson et al., 2004), and the FIRBACK survey (Puget et al., 1999; Dole et al., 2001) (see also reviews in Elbaz, 2005; Verma et al., 2005; Oliver \& Pozzi, 2005). The $15 \mu \mathrm{m}$ surveys (Elbaz et al., 2002) were particularly successful; the sources seen in these surveys could, with considerable extrapolation of the SED shape at longer wavelengths, account for around $80 \%$ of the CIB. Followup observations showed these sources have $\langle z\rangle \sim 0.8$, and that the comoving density of infrared light due to these $15 \mu \mathrm{m}$ sources is at least 40 times greater at $z \sim 1$ than in the local Universe (compare this to the B band

\footnotetext{
7 Though even at this stage source count models that could explain the CIB all invoked a population of high-redshift IR-luminous starbursts (Guiderdoni et al., 1998; Blain et al., 1999a; Rowan-Robinson, 2001; Xu et al., 2001)
} 
luminosity density, which is only about three times the local value at $z \sim 1)$. Not to be outdone, the FIRBACK survey found that the $170 \mu \mathrm{m}$ source counts show very strong evolution with redshift (reaching $\mathrm{z} \sim 1$ ), directly resolve $5 \%$ of the CIB (Dole et al., 2001), and are responsible for a dramatic rise in the integrated star formation rate, with a value at least ten times that seen locally at $z \sim 1$ (Rowan-Robinson et al., 1997; Flores et al., 1999; Pozzi et al., 2004). Overall, therefore, the ISO deep surveys put the IRAS discovery of strong IR galaxy evolution onto a very firm footing. Out to $\mathrm{z} \sim 1$ the ISO sources are chiefly LIRGs, not ULIRGs, and they are generally similar in many ways to lower redshift LIRGs, although possibly with lower average metallicity (Franceschini et al., 2003b; Elbaz, 2005; Liang et al., 2004).

The $170 \mu \mathrm{m}$ surveys also resulted in another interesting discovery; a large number of the $170 \mu \mathrm{m}$-selected objects have cooler dust temperatures and larger dust masses than those seen in starburst galaxies selected at mid-IR wavelengths. This is not unexpected for a long wavelength-selected survey, since a large luminosity from a cool dust component requires a larger dust mass than a warmer source. ISO however was the first observatory to show conclusively that large masses of 'cool' dust existed in many galaxies, from local spirals to distant ULIRGs. In local spirals this cold "cirrus" component is expected to be diffuse dust heated by the interstellar radiation field from later type stars. In ULIRGs however this cold component could alternatively be a compact dusty starburst with colder than average dust. This discovery has interesting implications for studies of ULIRGs at higher redshifts, as we will discuss in Section 5.3.

Focusing on ULIRGs; the largest ISO survey, and therefore the one most likely to discover ULIRGs in any number, was ELAIS (see Fig. 1). The most sensitive ELAIS band for LIRGs and ULIRGs was $15 \mu \mathrm{m}$, resulting in the detection of just under 100 ULIRGs, comprising $>10 \%$ of the $15 \mu \mathrm{m}$ sample. Around 10 of these are HLIRGs; the first of these to be identified being a QSO at $\mathrm{z}=1.01$ (Morel et al., 2001). These ULIRGs range in redshift up to $z>3$ and many are fit well in colour by an Arp 220-like SED. The longer wavelength ELAIS surveys result in many fewer sources due to decreased sensitivities; (Tavlor et al., 2005) have identified 4 likely ULIRGs in the FIRBACK-ELAIS N2 (FN2) $170 \mu \mathrm{m}$ population, while (Dennefeld et al., 2005) identify 1 or 2 in the FIRBACK-ELAIS N1 (FN1) field. (Saiina et al., 2003) identified a population of z 0.5-1 ULIRGs, representing $\sim 1 / 6$ of the total $170 \mu \mathrm{m}$ FN1 sample, and Chapman et al. (2002) identified two of these at $\mathrm{z}=0.5$ and 0.9 to be unusually cold systems with merger morphologies. Several sources in each field remained optically unidentified and are likely to be ULIRGs at moderate redshifts (0.5-1). Several ULIRGs and HLIRGs have been discovered in other ISO surveys, including a $z=1.7$ FeLoBAL QSO behind the $\mathrm{z}=0.56$ cluster J1888.16CL (Duc et al., 2002), and a radio detected ERO at $z=1.5$ (Pierre et al., 2001), probably a QSO with very hot dust.

Follow-up of $z \gtrsim 0.5$ ULIRGs revealed that many are involved in ongoing interactions, but that interestingly a significant number are QSOs with very massive $\left(>2 L^{*}\right)$ elliptical host galaxies (Farrah et al., 2002a). Detailed SED modeling (Rowan-Robinson \& Crawford, 1989; Rowan-Robinson, 2000; Verma et al., 2002; Farrah et al., 2002b) showed evidence for both starburst and AGN ac- 
tivity in most sources, with colossal implied star formation rates of up to $\sim 1000 M_{\odot} \mathrm{yr}^{-1}$. X-ray observations however found unexpectedly weak X-ray emission, implying either no obscured AGN or extremely high obscuration levels Wilman et al., 1998); the latter interpretation is favored by deeper observations which found evidence for Compton thick AGN in some (but not all) of these systems (Wilman et al., 2003). Very recently, Iwasawa et al. (2005) have used XMM to detect faint X-ray emission with a hint of the $6.4 \mathrm{keV} \mathrm{Fe} \mathrm{K} \alpha$ line from F15307+3252, which they attribute to a Compton thick AGN with $L(2$ $10 \mathrm{kev})>10^{45} \mathrm{erg} / \mathrm{s}$, which can account for a significant fraction of the infrared luminosity.

The picture that emerged from ISO, therefore, was one where very IRluminous galaxies become substantially more numerous with increasing redshift, making $z \sim 1$ LIRGs and ULIRGs a cosmologically significant population. The $0.5<z<1.0$ LIRG population seems quite similar to LIRGs at lower redshift, but also revealed a population of moderate redshift LIRGs with relatively cool dust emission. The higher redshift ULIRG population exhibits a more prevalent level of QSO activity than found in their local cousins, a result that could be either luminosity or redshift related since the most luminous ULIRGs are found at the higher redshifts.

\subsection{Resolving the CIB: ULIRGs at $z>1.5$}

The CIB had another startling surprise in store. Though ISO had proven itself remarkably adept at resolving much of the CIB, there remained a significant shortfall between the CIB as measured by COBE, and the sources detected by ISO. The background levels at $\lambda>200 \mu \mathrm{m}$ determined by FIRAS onboard COBE (Fixsen et al., 1998) implied a population of "colder" sources, probably $z \gtrsim 1$ systems with IR emission redshifted to longer wavelengths than the sources detectable in shallower, $\lambda<200 \mu \mathrm{m}$, ISO surveys.

Fortunately, the perfect instrument to search for just this kind of source had just been commissioned at the James Clerk Maxwell Telescope, namely the Sub-millimetre Common User Bolometer Array (SCUBA, Holland et al. 1999). SCUBA heralded the advent of bolometer arrays for sub-mm observations, and was one of the first sub-mm instruments that could map large areas of sky relatively quickly in the sub-mm; operating at substantially longer wavelengths than ISO, namely $850 \mu \mathrm{m}$ and $450 \mu \mathrm{m}$, SCUBA had the sensitivity to detect these distant 'cold' ULIRGs. Furthermore, the strongly negative k-correction at sub-mm wavelengths (Blain \& Longair, 1993) meant that a ULIRG at $z=5$ is (depending on the choice of cosmology) almost as easy to detect as a ULIRG of comparable luminosity at $z=1$. Other important sub-mm bolometer arrays include the $350 \mu \mathrm{m}$ optimized SHARC-II camera and the $1.1 \mathrm{~mm}$ Bolocam instrument, both at the Caltech Sub-mm Observatory, and the MAx-Planck Millimetre Bolometer (MAMBO) at the Institut de Radioastronomie Millimetrique (IRAM) $30 \mathrm{~m}$ telescope, which operates at $1.2 \mathrm{~mm}$.

The advent of sub-mm array instruments therefore prompted a plethora of surveys try to find these distant "cold" sources, ranging from ultra-deep sur- 
veys of lensing cluster fields Smail. Ivison \& Blain, 1997; Smail et al., 2002; Ivison et al., 2000) through deep blank-field surveys of small areas (Hughes et al., 1998; Barger et al., 1998), to wider area blank-field surveys to shallower depths (Eales et al., 2000; Scott et al., 2002; Borvs et al., 2003; Greve et al., 2004; Laurent et al., 2005). These surveys found a huge population of sub-mm bright, optically faint sources, with source counts of $320_{-100}^{+80}$ and $180 \pm 60$ per square degree at $S_{850}>10 \mathrm{mJy}$ (Scott et al., 2002). Taken together, the sources found in the various sub-mm \& mm surveys can directly account for around $50 \%$ of the CIB detected by FIRAS, and with reasonable extrapolation, can account for all of it (Barger et al., 1999b; Blain et al., 1999b).

The first step was to determine whether these sub-mm sources really are distant ULIRGs, or some other class of object. If, as is very plausible, the sub-mm emission from distant galaxies can be modeled with a simple modified blackbody, where the dust temperature distribution is characterised by $T$, then the flux at frequency $\nu, F(\nu)$, is given by $F(\nu) \propto \nu^{\beta} B(\nu, T)$, where $\beta$ is the spectral index (also referred to as the emissivity), and $B(\nu, T)$ is the Planck function. As a virtue of the shape of this function, sources with a redshift of $z \gtrsim 1$, a dust temperature of $\approx 25 \mathrm{~K}$, an $850 \mu \mathrm{m}$ flux of $S_{850}>1 \mathrm{mJy}$, and a reasonable choice of emissivity will, under most circumstances, be ULIRGs. Simple photometric redshift estimates (Bertoldi et al., 2000; Fox et al., 2002; Borvs et al., 2004; Webb et al., 2003) place virtually all the sources from sub-mm surveys at $z>1$, and most at $z>2$, meaning that the sub-mm sources are likely to be extremely luminous ULIRGs, with star formation rates substantially exceeding $1000 \mathrm{M}_{\odot} \mathrm{yr}^{-1}$. This implies that there are of order a few hundred ULIRGs per square degree at $z>1$, compared to about one ULIRG every four square degrees locally; though contributing less than $1 \%$ of the total extragalactic background light locally, ULIRGs were therefore shown to be a major, perhaps even dominant contributor at $z>1$.

Before proceeding further, there is an important cautionary note. A single sub-mm flux cannot be used to infer dust mass or source luminosity without assuming a dust grain size and temperature distribution (Hildebrand, 1983), thus, for example, large cold disks can be confused with compact warmer starbursts of very different bolometric luminosity (Kaviani. Haehnelt \& Kauffmann, 2003; Efstathiou \& Rowan-Robinson, 2003; Farrah et al., 2004a; Almaini et al., 2005). If the redshift is also unknown, a much lower luminosity foreground cold disk or even a Galactic dust cloud could easily mimic a high-redshift ULIRG (Lawrence, 2001). As we describe below, however, there is now good evidence that at least $\sim 70 \%$ of sub-mm sources are indeed $z>1$ ULIRGs, but within the remaining $30 \%$ there remains the possibility of significant sample contamination. Moreover, a strong temperature selection bias does exist in these very long-wavelength surveys; for example, a ULIRG at $2<z<3$ with $L_{i r} \sim 10^{13} L_{\odot}$ and a dust temperature of $60 \mathrm{~K}$ would not be detected in the wide, shallow surveys with SCUBA (Blain et al., 2004b). Such "hot" sources do appear to exist (Chapman et al., 2004b; Lutz et al., 2005), and could be responsible for up to $1 / 3$ of the DIRBE detected CIB. Therefore, sub-mm surveys alone do not necessarily provide a hyaline view of dust-shrouded star formation, or even of ULIRG 
activity, at high redshift. Spitzer is proving to be extremely important for discovering the warmest high-redshift ULIRGs (see Figure 1), as we shall describe further below.

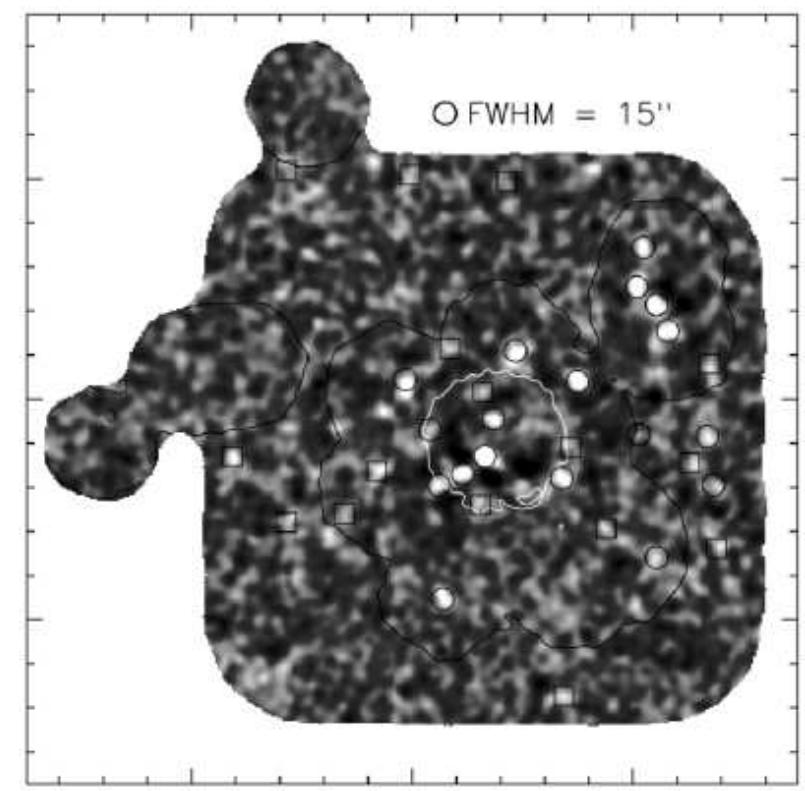

Fig. 4. An example of a recent submm survey; in this case the HDF-N SCUBA "Supermap" (Borvs et al., 2003). circles $=>4 \sigma$, squares $=3.5-4 D \sigma$. The white line encompasses the Hughes et al. (1998) SCUBA-HDF observations.

Due to the coarse angular resolution of SCUBA (and indeed most sub-mm \& $\mathrm{mm}$ observatories up to now) and the optical faintness of most of these sources, determining reliable ID's for follow-up proved to be extremely difficult (see Dunlop et al. 2004, for a perfect example). Nevertheless, since 1997, progress has slowly but surely been made in following up Sub-mm Galaxies (SMGs), and a clear picture of their nature has now started to emerge. A prime question is: how well do these distant systems resemble local ULIRGs? We would not necessarily expect them to be closely similar in nature because conditions are very different at high redshift, notably an earlier stage in the clustering evolution of the underlying dark matter, and larger gas fractions.

Optical and near-IR imaging (Smail et al., 1999; Webb et al., 2003; Smail et al., 2004; Pope et al., 2005) has shown that SMGs have a diverse range in optical properties, ranging from optically bright sources, to sources undetected in even the deepest optical imaging. Radio observations, particularly with the VLA, provided a major step forward in followup of SMGs (Ivison et al., 2002), detecting around $70 \%$ of the SMGs found in wide-field surveys with sufficiently high spatial resolution to allow reliable identification of optical/near-IR counterparts. Later, 
very high resolution radio studies have shown, intriguingly, that the radio emission in a surprisingly high fraction of SMGs, around $70 \%$, is spatially extended on scales of $\sim 10 \mathrm{kpc}$ (Chapman et al., 2004a), significantly larger than the dust emission seen in most local ULIRGs, which are generally less than a kpc across (Downes \& Solomon, 1998). The beam shape, especially when beam smearing is accounted for, is probably not well enough known to provide much evidence on the intrinsic shape of these radio sources, so possible explanations include large scale star formation over large disks, multiple compact starburst sites in a large disk or a coalescing group, or to AGN jet-induced star formation as seen in some distant radio galaxies (van Breugel et al., 2004). Another class of $\mathrm{z}>2$ large scale systems inferred to have ULIRG-level luminosities are the so-called Ly $\alpha$ blobs (Geach et al., 2005).

Early efforts to obtain spectroscopic redshifts for SMGs had been mostly stymied (but not entirely, see Barger et al., 1999a), not only by uncertain IDs and extreme optical faintness, but also because many SMGs lie within the so-called redshift 'desert', $1<z<2$, within which the bandpasses of most optical spectrographs could only sample weak absorption features, from which redshifts are hard to determine. But with high resolution radio ID's in hand, and significant tenacity, spectroscopic redshifts for SMGs were soon forthcoming (Chapman et al., 2005; Simpson et al., 2004). These surveys showed that SMGs have a median redshift of 2.4, removing any last lingering doubts that the (radio detected) SMGs were indeed high-redshift ULIRGs. The optical spectra themselves show evidence for both starbursts and AGN in most sources, high unobscured star formation rates, and that SMGs are generally metal rich (Swinbank et al., 2004). Very recent optical spectroscopic studies have focused on integral field spectroscopy, though instrumentation is only now becoming capable of integral field studies of distant SMGs. Early results however look very promising; the host galaxies of SMGs are metal rich, and appear to contain surprisingly large numbers of evolved stars and show evidence for ongoing interactions (Tecza et al., 2004; Swinbank et al., 2005).

With accurate redshifts it was possible to use heterodyne instruments (which generally have a very narrow bandpass, making spectroscopic redshifts essential for meaningful observations) to look at far-IR fine structure lines, particularly those of CO. CO surveys of SMGs (Genzel et al., 2003; Neri et al., 2003; Greve et al., 2005) revealed that they have enormous masses (often exceeding $\sim 1 \times 10^{11} M_{\odot}$ ) of molecular gas, usually unresolved but in some cases extended over 3-5kpc (Genzel et al., 2003), much greater than those masses seen in local ULIRGs and comparable to those seen in high-redshift radio galaxies. HST imaging shows that SMGs often possess disturbed morphologies, consistent with (but not solely supportive of) ongoing major mergers, similar to the local ULIRG population (Smail et al., 1998; Conselice et al., 2003). Very recent results based on deep X-ray observations have provided convincing evidence that the bulk of SMGs harbour a Compton thick AGN in addition to a starburst, implying that a sub-mm galaxy signposts a major period of growth of the central supermassive black hole in these systems, as well as an intense starburst (Alexander et al., 2005). There is some evidence that the periods of black hole growth and stellar 
mass buildup in these systems may not be coeval (Borvs et al., 2005), although there are uncertainties in the derivation of the X-ray obscuring columns, and therefore of the black hole mass (Polletta et al., 2006).

Further results have come from the first observations of SMGs with Spitzer. Results from IRAC and $24 \mu \mathrm{m}$ imaging (Egami et al., 2004; Fraver et al., 2004) have shown that SMGs have a wide range of observed mid-IR colours, and that their rest-frame mid-IR SEDs can in most cases be fitted with a starburst SED template, with the remainder being well fitted by a power-law AGN-dominated template. Photometric redshifts estimated from the near-infrared hump peaking at rest-frame $1.6 \mu \mathrm{m}$ and detected in the Spitzer IRAC bands $(3.6-8.0 \mu \mathrm{m})$ span $1<z<3.5$. Ivison et al. (2004) observed 9 MAMBO $1200 \mu \mathrm{m}$ sources in the same region, finding $75 \%$ of them to be starburst-dominated using Spitzer mid-infrared spectral shapes (assuming redshifts $\sim 2.5$ ), and concluding that the more AGN-like SEDs are consistent with AGN indications in these objects from UV/optical spectroscopy and X-ray imaging.

Spitzer will be a very powerful tool for finding large populations of ULIRGs at $z>1.5$, with many hundreds of candidates appearing in the Spitzer surveys such as SWIRE (Lonsdale et al. 2006), the Bootes Shallow field, the First Look Survey, the GTO deep surveys and GOODS (see Figure 1). Moreover Spitzer complements the cold dust selection function of the sub-mm and mm surveys with its mid-IR selection function and enhanced sensitivity to warm systems.

The first source counts and luminosity functions at $24 \mu \mathrm{m}, 70 \mu \mathrm{m}$ and $160 \mu \mathrm{m}$ from Spitzer extend the results seen by ISO. Spitzer is revealing large numbers of systems with significantly more extreme infrared/optical luminosity ratio than seen in the local Universe (Rowan-Robinson et al. 2005), a result also known from moderate redshift ISO surveys (Rowan-Robinson et al. 2004; Oliver \& Pozzi 2005). Rowan-Robinson et al. (2005) also find a significant population of very cool luminous Spitzer systems, which could be very large disks with quiescent star formation rates rather than starbursts. The $24 \mu \mathrm{m}$ counts in the GOODS fields (Papovich et al., 2004) show evidence for strong evolution, exceeding the 'no evolution' counts predictions by a factor of at least 10, and implying evolution in the comoving IR energy density of the form $(1+z)^{3.9 \pm 0.4}$ up to $z \sim 1$ (Le Flóch et al., 2005). The Spitzer counts at $70 \mu \mathrm{m}$ and $160 \mu \mathrm{m}$ (Dole et al., 2004) directly resolve $20 \%$ and $7 \%$ of the CIB at these wavelengths, respectively, and also show strong evolution, by a factor of $\sim 3$ over no evolution models, implying that the galaxies responsible for this background mostly lie in the redshift range $0.7<z<0.9$. Pérez-González et al. (2005) used find that LIRGs and ULIRGs are increasingly important contributors to the infrared energy density as redshift increases to $\mathrm{z} \sim 3$, being responsible for half of all star formation by $\mathrm{z} \sim 1.5$. The characteristic luminosity of the luminosity function, $L^{*}$, increases steadily with $\mathrm{z}$, consistent with the cosmic star formation rate density going as $(1+z)^{4}$ to $\mathrm{z}=0.8$, then flattening somewhat, and with ULIRGs playing a rapidly increasing role above $\mathrm{z}=1.3$. Le Flóch et al. (2005) came to similar conclusions out to $\mathrm{z} \sim 1$, and also compared the UV and IR star formation history since $\mathrm{z}=1$ using deep observations from Spitzer and GALEX, finding that the SFH has evolved much more strongly in the IR than in the UV, thus confirming the ISO- 
based results of Rowan-Robinson et al. (1997); Flores et al. (1999); Pozzi et al. (2004).

Yan et al. (2005) and Houck et al. (2005) have reported the first Spitzer low resolution IRS spectroscopy for extreme IR/optical z $\sim 2$ ULIRG candidates, demonstrating that Spitzer is capable of determining mid-infrared redshifts for the brightest mid-infrared galaxies $\left(f_{24}>0.75 \mathrm{mJy}\right)$. The space density of $L_{i r}>$ $10^{12.3} L_{\odot} \quad \mathrm{z} \sim 2$ Spitzer mid-infrared-selected ULIRGs may be similar to that of SMGs (Yan et al.) at this redshift, though, the sample sizes are as yet small and incomplete. Yan et al. infer a higher fraction of starburst-dominated ULIRGs in their sample than Houck et al., which is probably due to the additional selection criterion on high $24 / 8 \mu \mathrm{m}$ colour ( $i e$ a rejection of mid-IR warm sources). Lutz et al. (2006) report a low $1.2 \mathrm{~mm}$ detection rate these systems using MAMBO, and suggest that their mid-infrared-selected sample may have significantly warmer dust than submm-selected samples. Franceschini et al. (2004), Martinez-Sansigre et al. (2005), Donley et al. (2005) and Polletta et al. (2006) have used Spitzer surveys to discover extensive populations of heavily obscured AGN populations that can have very high X-ray obscuring columns, including two z $>2$, Comptonthick SWIRE HLIRGs with AGN torus-dominated mid-IR SEDs (Polletta et al., 2006). These new Spitzer results indicate that in many ULIRGs highly obscured QSOs are extremely difficult to detect at any wavelength, including hard X-ray and mid-infrared, due to extreme optical depths.

Daddi et al. (2005) have reported the first Spitzer results from the Great Observatories Origins Deep Survey (GOODS) for $\mathrm{z} \sim 2$ ULIRGs, finding that typical massive $\left(M_{\text {stellar }} \sim 10^{11} M_{\odot}\right)$ star forming galaxies at this redshift are ULIRGs, based on $24 \mu \mathrm{m}$ and radio detections, with a co-moving space density of ULIRGs at $\mathrm{z}=2$ at least 3 orders of magnitude greater than the local one.

In summary it would be fair to say that surveys with SCUBA and other sub-mm/mm observatories, and their followup, have revolutionized our perceptions of ULIRGs, while Spitzer stands poised to contribute its own revolution of knowledge about them. From being little more than an interesting oddity in the local Universe, ULIRGs have become a crucially important population at $z>1$. Furthermore, whilst $z>1$ ULIRGs are similar in many ways to their local counterparts - both populations apparently being heavily dust-obscured starbursts and/or AGN triggered by interactions between large, evolved systems - there are important differences. High-redshift ULIRGs are substantially more gas rich than their local counterparts, and some may have extended rather than compact starbursts. Moreover the star formation rates implied (if AGN do not strongly dominate the energetics) are enormous, pushing the limits on theoretical ideas for a "maximal" starburst in a massive galaxy: $\mathrm{M}_{\text {aas }} / t_{d y n}=10^{11} \mathrm{M}_{\odot} / 10^{8}$ yrs $\sim 1000 \mathrm{M}_{\odot} /$ yr (Eggen. Lvnden-Bell \& Sandage, 1962). To understand these differences, and place these high-redshift ULIRGs into a cosmological context, we must turn to theories of galaxy and large-scale structure formation. 


\section{ULIRGs and Large-scale structure}

The observation that these distant ULIRGs appear to be very massive systems means that large amounts of raw material are needed to make one, implying that ULIRGs are likely to be found in regions with large concentrations of baryons. Therefore, in order to understand when, where and why ULIRGs exist, we must turn to galaxy and large-scale structure formation models to see where such large concentrations of baryons are predicted to be found. Modern structure formation models describe the evolution of the total mass distribution (comprising both non-baryonic and baryonic 'cold' dark matter) via the evolution of initially Gaussian density fluctuations, which means that the evolution of the total mass distribution should be traced by the formation and evolution of galaxies in some determinable way. Density fluctuations in the dark matter distribution, commonly referred to as 'halos', are predicted to undergo successive mergers over time to build halos of steadily increasing mass. Galaxies are predicted to form from the baryonic matter in these halos, driven either by inter- or intra-halo mergers, or passive collapse of baryons to the halo central regions, or some combination of all three mechanisms (Meza et al., 2003; Kobavashi, 2004). This framework of 'biased' hierarchical buildup (Cole et al., 2000; Granato et al., 2000; Hatton et al., 2003), coupled with a $\Lambda$ cosmology (Spergel et al., 2003), has proven to be remarkably successful in explaining many aspects of galaxy and large-scale structure formation.

Within this framework, the largest concentrations of baryons are expected to be found within the most massive dark matter halos. A basic verification of this idea can be seen in the local Universe, where the most massive elliptical galaxies are almost invariably found within very rich galaxy clusters; which presumably correspond to large overdensities of baryons in the local Universe. The formation history of these very massive galaxies, even before the advent of sub-mm surveys, was controversial. The 'naive' expectation from hierarchical theory would be that massive galaxies form relatively late and over a long period of time, representing successive mergers between dark matter halos, building up the required large baryon reservoirs (Baugh et al., 1998), and indeed some massive galaxies do appear to form in this way (van Dokkum et al., 1999; Tamura \& Ohta, 2003: Bell et al., 2004). There is however observational evidence that many massive galaxies may form at high redshift and on short timescales, for example from the discovery of evolved systems at high redshift (Dunlop et al., 1996; Martini, 2001; Blakeslee et al., 2003), the extremely tight K-band Hubble relation for radio galaxies (De Breuck et al., 2002) implying a very rapid early formation of the most massive galaxies (Rocca-Volmerange et al., 2004), and an inferred early star formation epoch from the colour-magnitude diagram in clusters at $z=0.5$ (Ellis et al., 1997), implying that the stars in very massive local galaxies formed within a few Gyr of each other at $z>1$.

The SMGs and Spitzer high z ULIRG samples appear to be perfect candidates for the formation events for those local massive ellipticals that appeared

to form very early and very fast; they lie at $z \simeq 2.5$ (Simpson et al., 2004; Chapman et al., 2005), which is a high enough redshift to make stars with the 
ages seen locally, their star formation rates are high enough to make all the stars in a local massive elliptical fast enough to satisfy the observed colour-scaling relationships, and their comoving number density is comparable to that of local $\gtrsim 3 L^{*}$ ellipticals (Scott et al., 2002; Chapman et al., 2004b). Serious theoretical difficulties remain however. Early CDM-based galaxy formation models lacked the detailed astrophysics to explain the number of observed SMGs and their star formation rates (Granato et al., 2000; Somerville. Primack \& Faber, 2001; Guiderdoni et al., 1998). Later models included improved treatments of starbursts, but still had to include further modifications to explain the observed sub-mm counts. These include a top-heavy initial mass function for the stars formed in bursts, so that the same sub-mm flux can be produced with lower star formation rates (Baugh et al., 2005) ${ }^{8}$, modified treatments of virialization and the survival of subhalos to solve the 'overmerging' problem in the standard PressSchechter formalism and make the SFRs high enough (van Kampen et al., 1999, 2005), and modifications to gas cooling and supernova feedback (Granato et al., 2004).

Fundamentally, there are two testable basic predictions of these hierarchical models for high-redshift ULIRGs. Firstly, these ULIRGs should reside within rich environments, that is to say we should see clustering of other galaxies around the ULIRGs on length scales of $<5 \mathrm{Mpc}$, representing galaxy overdensities resulting from the local overdensity of baryons. Testing this observationally is, however, difficult. At $z<1$ there have been numerous successful measurements of galaxy overdensities around AGN using deep optical/near-IR imaging to find evolved systems (Wold et al., 2001, 2003), but at the mean redshift of high-redshift ULIRGs it is certainly not clear whether their local environments have built up overdensities of evolved galaxies, even if they are overdense in baryons. Secondly, we should observe the high-redshift ULIRGs themselves to cluster together strongly on the sky, or in other words clustering of just the high-redshift ULIRGs on length scales $\underset{\sim}{2} 5 \mathrm{Mpc}$. This prediction is a consequence of the assumed form of the evolution of density fluctuations with redshift; rare fluctuations in the underlying mass distribution are predicted to cluster together on the sky, with the strength of clustering depending on the degree of rarity (Bardeen et al., 1986). Very massive halos (those with masses $\gtrsim 10^{14} M_{\odot}$ ), which are universally predicted to be extremely rare at high redshift, are therefore predicted to cluster together very strongly (e.g. Moscardini et al. 1998; Kauffmann et al. 1999; Mo \& White 2002). This clustering should be traced by very massive galaxies, as only the most massive halos contain sufficient baryon reservoirs to form these galaxies. This also means that the clustering strength of massive galaxies should translate straightforwardly to measure of halo mass (Percival et al., 2003). Strong clustering among high-redshift ULIRGs is also predicted if, as some suspect, they are part of a population uniting QSOs and spheroids (Magliocchetti et al., 2001).

\footnotetext{
${ }^{8}$ an interesting conundrum from this model is that while the sub-mm counts are predicted to be dominated by bursts, these bursts would be responsible for making only a small fraction of the stellar mass in evolved ellipticals in the local Universe if their IMFs are top heavy.
} 
Measuring this form of clustering observationally is however also difficult, requiring either $>50$ sources with spectroscopic redshifts so that 'associations' in redshift space between different sources can be searched for, or a minimum of several hundred sources with a reasonably well constrained redshift distribution, and found over a large enough area of sky such that the length scales of interest can be examined.
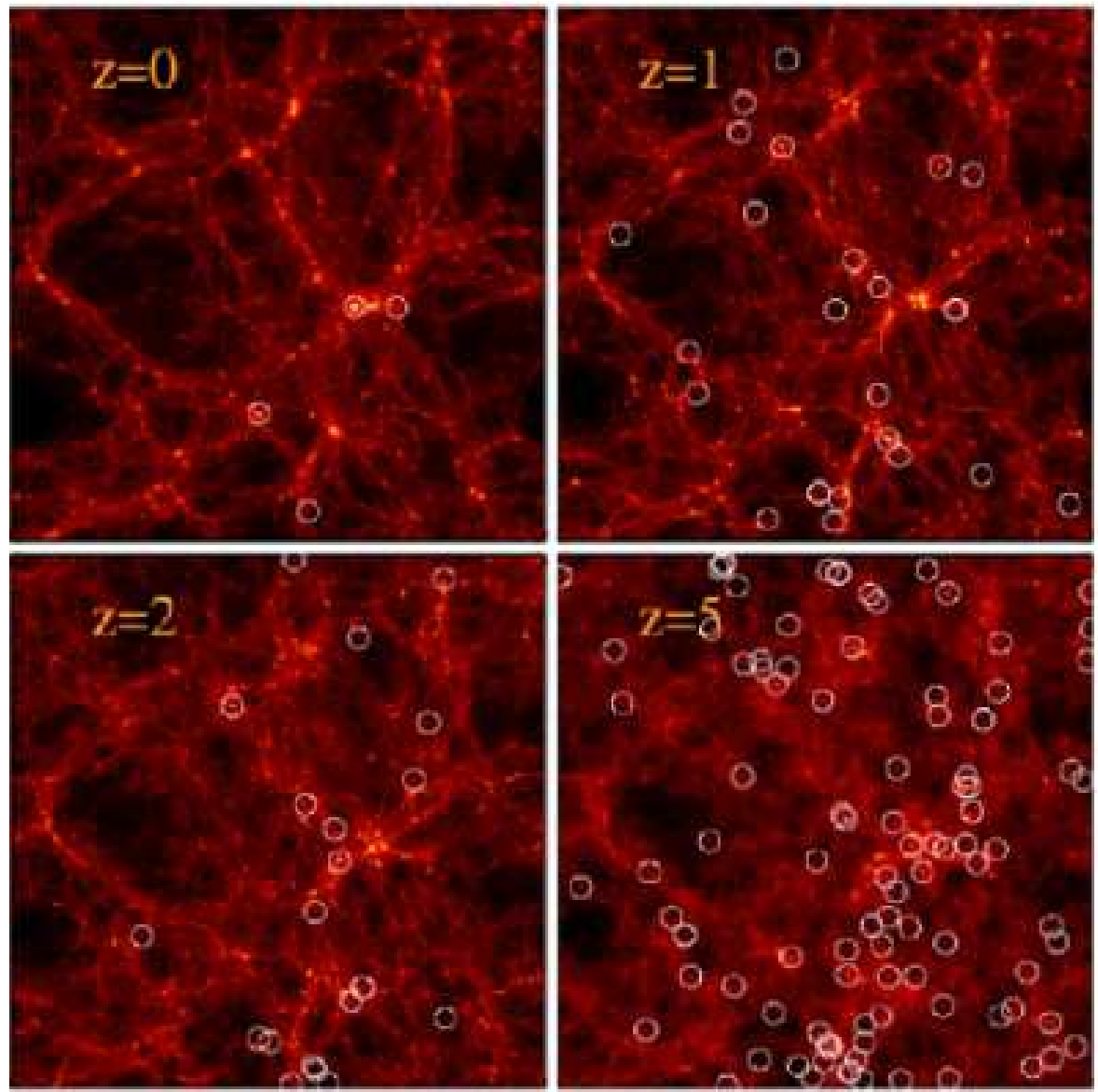

Fig. 5. An example of the cosmological hydrodynamic $1 \mathrm{CDM}$ simulation (SPH G6 run) described in Nagamine et al. (2005a,b). Each panel has a comoving size of $143 \mathrm{Mpc}$ on a side, and the starforming galaxies with instantaneous $\mathrm{SFR}>100 \mathrm{M}_{\odot} / \mathrm{yr}$ at each epoch are indicated by the circles (K. Nagamine, priv. comm.)

Despite these difficulties, numerous studies of both the environments of highredshift ULIRGs, and their clustering, have taken place, and while these studies are not (yet) conclusive, they do generally lend support to the idea that high-redshift ULIRGs form within massive dark matter halos. Considering envi- 
ronment studies first; near-IR imaging studies of the environmental richness of ULIRGs over $0.5<z<1.5$ (Farrah et al., 2004b) have shown that these systems reside in richer environments than their local counterparts on average, and that some reside in moderate rich clusters even at $z \gtrsim 1$, though it is not clear whether this represents a genuine increase in environmental richness for ULIRGs, or whether this just reflects the global increase in 'bias' over $0<z<1$. At lower redshifts, LIRGs and ULIRGs have also been found in clusters (Duc et al., 2004; Lemonon et al., 1998; Kleinmann et al., 1988). Turning to higher redshifts, then several authors have noted overdensities of sub-mm sources around highredshift systems that are thought to reside within mass overdensities, including $z \sim 4$ radio galaxies (Stevens et al., 2003; De Breuck et al., 2004) (several of these sub-mm sources are also detected in the X-ray, suggesting the presence of obscured AGN (Smail et al., 2003) and seen as $z \sim 3$ Lyman Break galaxies (Chapman et al., 2001; Geach et al., 2005).

Turning to high-z ULIRG clustering; early efforts to measure angular clustering on the sky came up against the limitations of available instrumentation; at the time of writing there exists no sub-mm array instrument that can map large enough areas of the sky to the required depths (though SCUBA2, APEX and Herschel, are coming soon). No clear picture has therefore emerged; some sub-mm surveys have uncovered tentative hints of clustering on scales of a few arcminutes (Scoville et al., 2000), whereas others show no signs of clustering whatsoever (Borvs et al., 2003). An intriguing result from these wide field surveys is that the high-redshift ULIRGs appear to trace moderately bright X-ray survey sources on the sky, even though the direct overlap between the two populations is minimal (Almaini et al., 2003). This could suggest that the high-redshift sub$\mathrm{mm}$ and $\mathrm{X}$-ray populations are tracing the same, overdense dark matter halos. More recent efforts to measure SMG clustering using spectroscopic redshifts have met with greater success, resulting in a reliable detection of clustering for the first time (Blain et al., 2004a). The strength of the clustering, while significant, is not particularly strong, at $r_{0}=6.9 \pm 2.2$ (comoving). This clustering strength is consistent with high-redshift ULIRGs residing in $\sim 10^{13} M_{\odot}$ halos which will eventually become the cores of rich clusters in the local Universe, but the relatively high redshift of the sources in this sample, coupled with the significant error on their clustering and the unknown growth modes of dark matter overdensities (see e.g. Matarrese et al. 1997; Moscardini et al. 1998) leaves other possible interpretations open. More precise measurements of both high-redshift ULIRGs, and other ULIRGs at lower (and higher) redshifts are needed to resolve this issue. The new Spitzer ULIRG surveys, sampling much larger volumes and linear dimensions than current submm/mm surveys, can be expected open up this field of enquiry dramatically.

\section{Open Questions and New Directions}

An incredible amount of energy has been spent trying to understand ULIRGs in the decade since the Sanders \& Mirabel (1996) review, and this review has 
not been able to do adequate justice to all this effort. To summarize the current state of knowledge we address the a few of the most pressing questions from our own perspective.

In the relatively local Universe, ULIRGs are almost all associated with major mergers of gas rich systems. Evolutionary schemes whereby ULIRGs transition into optical QSOs at the end stages of a merger, with an elliptical as the end result, are highly attractive and well supported by simulations. The reality is obviously complex, however, depending strongly on the details of the merger encounter, in particular bulge mass. Observationally it is not necessarily the case that all ULIRGs represent late stages in the merger sequence, however the most luminous systems do seem to represent the late stages, implying that it requires the extraordinary high density conditions of the final merger stages to produce these tremendous levels of power. Evidence that some optical QSOs may have more massive hosts than ULIRGs, or inhabit richer environments, would indicate that the true situation is more complex, but matching AGN/ULIRG samples for such studies is very challenging.

Merger sequences connect starbursts and AGN tightly together via the availability of fuel to build the stellar and SMBH masses, and to fuel the AGN. AGN orientation schemes also tie ULIRGs and QSOs tightly together since off-axis QSOs will be obscured and may appear as ULIRGs. Therefore the empirical determination of the relative power of AGN and starbursts for both infraredselected ULIRGs and optically-selected ULIRG QSOs is a paramount question. Most ULIRGs are thought to be powered mostly by starbursts however indicators of AGN activity increase with luminosity, recognised from near- and mid-infrared spectroscopy, X-ray detections or VLBI radio imaging. All of these indicators are tricky however and, because nuclear (Starburst) and gravitational accretion (AGN) power often compete for dominance we need more robust estimators of the SFR and AGN activity to compare with merger stage, morphology, mass, etc. to disentangle an evolutionary sequence from other effects. For example, low/high-resolution mid-IR spectroscopic indicators can be obscured; Spitzer will do an excellent job of measuring diagnostic features from $5-40 \mu \mathrm{m}$, but interpretation will require an understanding of extinction as a function of wavelength throughout the spectrum as well as modeling with full radiative transfer treatment. X-ray columns can be high enough to severely limit AGN detection; separating soft (starburst or reflected AGN) vs hard (AGN or X-ray binary) emission will require sensitivity at higher energies with Con-X and XEUS. High-resolution radio imaging has to be capable of mapping at brightness temperatures above $10^{6} \mathrm{~K}$ to distinguish jet structures from RSN complexes, because starburst and radio-quiet AGN emission cannot be distinguished on the basis of power alone. But we do not yet even understand the complex radio supernovae in the one, closest, well studied system, Arp 220, where questions remain about the nature of the LRSN, the IMF in the luminous starburst, etc. Such imaging observations are time consuming, but need to be expanded to much larger samples. One possible line of inquiry may be to obtain $\mathrm{BH}$ mass estimates from the molecular gas kinematics with ALMA $(r \sim 20 \mathrm{pc})$ as has been done for NGC 4258 in $\mathrm{H}_{2} \mathrm{O}$. 
Other questions remain, eg.: What are the compact masers telling us? Are they pointers to AGN/mass concentrations?

ULIRGs increase dramatically in importance with redshift, from being a very minor component at $\mathrm{z} \sim 0$ to possibly dominating the energy density at $\mathrm{z}>2$, and ULIRG populations at $\mathrm{z}>2$ are much more extreme beasts than at low redshift, with larger IR luminosities, gas masses and, in some cases, larger ratios of infrared-to-optical luminosity than local ULIRGs, by factors of 10 to 100 . They also show an increase in space density of a factor of 100-1000 (Chapman et al., 2005; Le Flóch et al., 2005; (Pérez-González et al., 2005), though this number is very uncertain because the low and high redshift LFs barely overlap in luminosity range; at low redshift the number density of HLIRGs is intrinsically extremely small, while at high redshifts, submm surveys are only sensitive only to systems above a few $\times 10^{12} L_{\odot}$. Wide area Spitzer luminosity functions can be expected to bridge this gap (Lonsdale et al. 2006b, Babbedge et al. 2006). There is clear evidence that at high redshift many ULIRGs are triggered by mergers of gas rich systems, but there are also some striking differences from local systems: extended radio emission and some evidence for very large cool disk systems, which may not even qualify as starbursts, but rather star formation ocurring in a more-or-less quiescent fashion in these very large disks.

As noted above, the incidence of AGN seems to increase with luminosity, and this is also a strong redshift effect since the most luminous systems are only known at higher redshifts. As also noted above, it remains remarkably difficult to determine the relative power contribution from star formation vs. AGN activity in many ULIRGs. Many lines of argument are used to conclude that star formation powers most ULIRGs at all redshifts. These include the energy reservoir available from nucleosynthesis relative to SMBH accretion; low AGN power deduced from absorption-corrected hard X-ray detections; the number of obscured AGN required to explain the XRB and the correlation between bulge and SMBH masses which suggest bulge growth events must accompany SMBH growth. Arguments for a stronger importance for AGN power include that luminosities above $10^{13} L_{\odot}$ are very extreme for a starburst event while many high-redshift QSOs are known with such luminosities; a large number of QSOpowered ULIRGs is expected in AGN unification-by-orientation schemes; hard X-ray absorption correction is difficult, and Compton thick AGN such as Mrk 231 are very difficult to detect at high redshift.

Perhaps one of the strongest lines of evidence one way or the other comes from the impressive correlation between warm dense molecular gas traced by the HCN molecule with infrared luminosity (Gao \& Solomon, 2004), which extends across 4 orders of magnitude in luminosity from $10^{9}$ to $10^{13}$. There's little doubt that low luminosity systems are powered by disk star formation, so this relation provides support for the picture that even HLIRGs can be powered by star formation. HCN ULIRG detections are limited at high luminosity/redshift, however, and they tend to be displaced above the lower luminosity redshift relation towards excess $L_{I R} / M_{H C N}$ (Carilli et al., 2005), which is consistent with the presence of an an additional, AGN, power source but is also within the scatter of the lower redshift starburst relation. Spitzer will be able to provide deep 
insights into this debate because the presence of a significant AGN power source will be evident the mid-infrared spectral shape, as demonstrated by the recent flurry of papers reporting the discovery of large populations of highly obscured QSO/ULIRGs (Martinez-Sansigre et al., 2005; Polletta et al., 2006; Yan et al., 2005; Houck et al., 2005; Donlev et al., 2005).

The causes for the dramatic differences in ULIRG number density and nature since $\mathrm{z} \sim 3$ must lie with the evolving matter density field and galaxy formation processes. Since interactions and mergers are clearly implicated in most ULIRG activity, the most natural assumption to make is that these objects follow 'the action'. They are, after all, the most dramatic active events that occur in the Universe, the prima donnas of the show. At a given epoch they are likely to occur where the most dramatic gas rich mergers are happening, as illustrated in the simulation of K. Nagamine (private communication) in Figure 5 At the earliest epochs of galaxy formation the action is at the sites of future rich clusters, while at current times loose groups are ideal future ULIRG sites since there are a few close galaxies with moderate relative velocity, and gas has not been exhausted yet. At intermediate redshifts, the best ULIRG sites may be the interaction zones in cluster-cluster mergers. These ideas are consistent with the sparse currently available observations of ULIRG environments at various redshifts.

So what are the key unanswered questions and where do we go from here to answer them? So far ULIRG samples are quite sparse - numbering in the hundreds over a huge redshift range. We clearly need much larger, volume-limited samples. Moreover there are serious selection biases since high-redshift systems have been selected at sub-mm wavelengths by their cool dust, and substantially warmer systems will have been missed. Therefore we need to understand the full range of ULIRG SEDs and obtain complete samples with well measured SEDs via selection from the mid-IR to the sub-mm. Spitzer will clearly revolutionize this field, bringing in the warm-samples to complement the sub-mm ones, but also selecting PAH-dominated objects as the mid-IR PAH features redshift into the $24 \mu \mathrm{m}$ band. Larger, deeper sub-mm \& $\mathrm{mm}$ surveys are also required to reach down the luminosity function at $\mathrm{z}>1$ and combat the effects of cosmic variance.

To complement the ULIRG samples it's obviously important to study complete QSO samples in the same volumes of space and to determine the lifetimes of both types of object, to directly investigate their evolutionary relationships. A tricky aspect to this work will be the fact that ULIRG and QSO luminosities are predicted by the most recent simulations to vary very dramatically on short timescales (as are SMBH and stellar masses), especially as the SMBH building and AGN accretion phase accelerates, so matching samples by luminosity or mass is probably going to match apples and oranges.

Key to understanding the role of ULIRGs in galaxy formation - their connections to other kinds of systems, their progenitors and their descendents, the connections between bulge and SMBH building and the relative importance of starburst vs quiescent star formation modes - is to determine the host galaxy properties of ULIRGs and QSOs, especially stellar masses and morphologies, and to determine the richness of their environments and their clustering properties as a function of redshift. 
The future holds great promise for answering these questions. Measuring the clustering of ULIRGs requires wide area far-IR/sub-mm surveys, which are or will be forthcoming from Spitzer, SCUBA-2, APEX, ASTRO-F, Herschel and WISE. These surveys will also provide the large samples of ULIRGs needed to measure the range in dust temperatures in the distant ULIRG populations, with detailed followup provided by other groundbased facilities. Measuring sizes, morphologies, and stellar masses, assessing the impact of gravitational lensing and determining environmental richness, on the other hand, require deep pointed observations in the near-IR to radio. Here the key facilities are Spitzer, JWST, ALMA, the EVLA and SKA. Imaging in molecular and other submm/mm lines will also be possible with ALMA.

\section{Acknowledgements}

We are deeply indebted to Colin Borys, Jason Surace, Kentaro Nagamine, C. Kevin Xu \& Lee Armus who provided original figures from their recent work for this review. The Spitzer IRS ULIRG team who responsible for the spectra shown and described in Section 3 include B. Thomas Soifer, James Houck, Lee Armus, Vassilis Charmandaris, Henrik Spoon, Jason Marshall, Jeronimo Bernard-Salas \& Sarah Higdon. CJL and HES are indebted to Yu Gao and the organizers of the Lijiang Workshop on "Extreme Starbursts" which contributed greatly to the focus of this review.

\section{References}

Alexander, D. M., et al. 2005, Nature, 434, 738

Almaini, O., et al. 2003, MNRAS, 338, 303

Almaini, O., Dunlop, J. S., Willott, C. J., Alexander, D. M., Bauer, F. E., \& Liu, C. T. 2005, MNRAS, 358, 875

Armus, L., Heckman, T., \& Miley, G. 1987, AJ, 94, 831

Armus, L., Heckman, T., Miley, G. 1988, ApJ, 326, L45

Armus, L., et al. 2004, ApJS, 154, 178

Armus, L., et al. 2005, in preparation

Baan, W. A., Wood, P. A. D., \& Haschick, A. D. 1982, ApJL, 260, L49

Baan, W. A. 1989, ApJ, 338, 804

Baan, W. A., Haschick, A. D., \& Henkel, C. 1989, ApJ, 346, 680

Babbedge, T. S. R., Rowan-Robinson, M., Lonsdale, C. J., et al. 2006, MNRAS, submitted

Baldwin, J. A., Phillips, M. M., \& Terlevich, R. 1981, PASP, 93, 5

Balestra, I., Boller, Th.,Gallo, L., Lutz, D., \& Hess, S. 2005, A\&A, in press (astro-ph/0507321)

Bardeen, J. M., Bond, J. R., Kaiser, N., \& Szalay, A. S. 1986, ApJ, 304, 15

Barger, A. J., et al. 1998, Nature, 394, 248 
Barger, A. J., Cowie, L. L., Smail, I., Ivison, R. J., Blain, A. W., \& Kneib, J.-P. 1999a, AJ, 117, 2656

Barger, A. J., Cowie, L. L., \& Sanders, D. B. 1999b, ApJL, 518, L5

Barger, A. J., et al. 2005, AJ, 129, 578

Barnes, J. E. 1989, Nature, 338, 123

Barnes, J. E., \& Hernquist, L. 1992, ARA\&A, 30, 705

Barnes, J. E., \& Hernquist, L. 1996, ApJ, 471, 115

Baugh, C. M., Cole, S., Frenk, C. S., \& Lacey, C. G. 1998, ApJ, 498, 504

Baugh, C. M., et al. 2005, MNRAS, 356, 1191

Beichman, C. A., et al. 1986, ApJL, 308, L1

Bell, E. F., et al. 2004, ApJ, 608, 752

Bertoldi, F., et al. 2000, A\&A, 360, 92

Blain, A. W. \& Longair, M. S. 1993, MNRAS, 264, 509

Blain, A. W., Smail, I., Ivison, R. J., \& Kneib, J.-P. 1999a, MNRAS, 302, 632

Blain, A. W., Kneib, J.-P., Ivison, R. J., \& Smail, I. 1999b, ApJL, 512, L87

Blain, A. W., Chapman, S. C., Smail, I., \& Ivison, R. 2004a, ApJ, 611, 725

Blain, A. W., Chapman, S. C., Smail, I., \& Ivison, R. 2004b, ApJ, 611, 52

Blakeslee, J. P., et al. 2003, ApJL, 596, L143

Boller, T., Bertoldi, F., Dennefeld, M., \& Voges, W. 1998, A\&AS, 129, 87

Boller, T., Gallo, L. C., Lutz, D., \& Sturm, E. 2002, MNRAS, 336, 1143

Bondi, M., Pérez-Torres, M.-A., Dallacasa, D., \& Muxlow, T. W. B. 2005, MNRAS, 575

Borne, K. D., Bushouse, H., Lucas, R. A., \& Colina, L. 2000, ApJL, 529, L77

Borys, C., Chapman, S., Halpern, M. \& Scott, D. 2003, MNRAS, 344, 385

Borys, C., Scott, D., Chapman, S., Halpern, M., Nandra, K., \& Pope, A. 2004, MNRAS, 355, 485

Borys, C., et al. 2005, ApJ, in press (astro-ph/0507610)

Braito, V., et al. 2004, A\&A, 420, 79

Bryant P. \& Scoville, N. 1999, AJ, 117, 2632

Burbidge, G. R. \& Stein, W. A. 1970, ApJ, 160, 573

Bushouse, H. A., et al. 2002, ApJS, 138, 1

Canalizo, G. \& Stockton A. 2001, ApJ, 555, 719

Carilli, C. L., Wrobel, J. M., \& Ulvestad, J. S. 1998, AJ, 115, 928

Carilli, C. L., et al. 2005, ApJ, 618, 586

Cesarsky, C. J., et al. 1996, A\&A, 315, L32

Chapman, S. C., Lewis, G., Scott, D., Richards, E., Borys, C., Steidel, C., Adelberger, K., \& Shapley, A. 2001, ApJL, 548, L17

Chapman, S. C., Smail, I., Ivison, R. J., Helou, G., Dale, D. A., \& Lagache, G. 2002, ApJ, 573, 66

Chapman, S. C., Smail, I., Windhorst, R., Muxlow, T., \& Ivison, R. J. 2004a, ApJ, 611, 732

Chapman, S. C., Smail, I., Blain, A. W., \& Ivison, R. J. 2004b, ApJ, 614, 671

Chapman, S. C., Blain, A. W., Smail, I. \& Ivison, R. J. 2005, ApJ, 622, 772

Chary, R., et al. 2004, ApJS, 154, 80

Clavel, J., et al. 2000, A\&A, 357, 839

Clegg, P. E., et al. 1996, A\&A, 315, L38 
Clements, D. L., Sutherland, W. J., McMahon, R. G., \& Saunders, W. 1996, MNRAS, 279, 477

Clements, D. L., et al. 2002, ApJ, 581, 974

Cole, S., Lacey, C. G., Baugh, C. M., \& Frenk, C. S. 2000, MNRAS, 319, 168

Colina, L., et al. 2001, ApJ, 563, 546

Colina, L., Arribas, S., \& Clements, D. 2004, ApJ, 602, 181

Condon, J. J., Huang, Z.-P., Yin Q. F. \& Thuan, T. X. 1991, ApJ, 378, 65

Conselice, C. J., Chapman, S. C., \& Windhorst, R. A. 2003, ApJL, 596, L5

Cutri, R., Huchra, J., Low, F., Brown, R., \& vanden Bout, P. 1994, ApJL, 424, L65

Cutri, R., Nelson, B., Francis, P. \& Smith, P. in "AGN Surveys", ASP Conf. Serire, 284, 127

Daddi, E., et al. 2005, ApJ, 626, 680

Darling, J., \& Giovanelli, R. 2002, AJ, 124, 100

De Breuck, C., van Breugel, W., Stanford, S. A., Röttgering, H., Miley, G., \&

Stern, D. 2002, AJ, 123, 637

De Breuck, C., et al. 2004, A\&A, 424, 1

de Graauw, T., et al. 1996, A\&A, 315, 49

de Grijp, M. H. K., Miley, G. K., Lub, J. \& de Jong, T. 1985, Nature, 314, 240

de Jong, T., Klein, U., Wielebinski, R. \& Wunderlich, E. 1985, A\&A, 147, L6

Dennefeld, M., et al. 2005, A\&A, in press (astro-ph/0504344)

Di Matteo, T., Springel, V. \& Hernquist, L. 2005, Nature, 433, 604

Diamond, P. J., Norris, R. P., Baan, W. A., \& Booth, R. S. 1989, ApJL, 340, L49

Diamond, P. J., Lonsdale, C. J., Lonsdale, C. J., \& Smith, H. E. 1999, ApJ, 511, 178

Dole, H., et al. 2001, A\&A, 372, 364

Dole, H., et al. 2004, ApJS, 154, 87

Donley, J., Rieke, G., Rigby, J. \& Perez-Gonzalez 2005, ApJ, in press (astro-ph/0507676)

Dopita, M. A., Kewley, L. J., Heisler, C. A., \& Sutherland, R. S. 2000, ApJ, 542, 224

Dopita, M. A., Groves, B. A., Fischera, J. \& Sutherland, R. S. 2005, ApJ, 619, 755

Downes, D., \& Solomon, P. M. 1998, ApJ, 507, 615

Dubinski, J., Mihos, J. C., \& Hernquist, L. 1999, ApJ, 526, 607

Duc, P.-A., et al. 2002, A\&A, 389, L47

Duc, P., et al. 2004, IAU Colloq. 195: Outskirts of Galaxy Clusters: Intense Life in the Suburbs, 347

Dunlop, J. S., et al. 1996, Nature, 381, 581

Dunlop, J. S., et al. 2003, MNRAS, 340, 1095

Dunlop, J. S., et al. 2004, MNRAS, 350, 769

Dwek, E., et al. 1998, ApJ, 508, 106

Eales, S., et al. 2000, AJ, 120, 2244

Efstathiou, A. Rowan-Robinson, M. 2003, MNRAS, 343, 322

Egami, E., et al. 2004, ApJS, 154, 130 
Eggen, O., Lynden-Bell, D. \& Sandage, A. 1962, ApJ, 136, 748

Elbaz, D., et al. 2002, A\&A, 384, 848

Elbaz, D. 2005, to be published in 'ISO science legacy - a compact review of ISO major achievements', Space Science Reviews, Springer

Ellis, R. S., et al. 1997, ApJ, 483, 582

Elston, R., Cornell, M. E., \& Lebofsky, M. J. 1985, ApJ, 296, 106

Elvis, M. 2000, ApJ, 545, 63

Fabian, A. C. 1999, MNRAS, 308, L39

Farrah, D., et al. 2001, MNRAS, 326, 1333

Farrah, D., Verma, A., Oliver, S., Rowan-Robinson, M. \& McMahon R. 2002a, MNRAS, 329, 605

Farrah, D., Serjeant, S., Efstathiou, A., Rowan-Robinson M. \& Verma A. 2002b, MNRAS, 335, 1163

Farrah, D., et al. 2003, MNRAS, 343, 585

Farrah, D., et al. 2004a, MNRAS, 349, 518

Farrah, D., Fox, M., Rowan-Robinson, M., Clements, D., \& Afonso, J. 2004b, ApJ, 603, 489

Farrah, D., Surace, J. A., Veilleux, S., Sanders, D. B., \& Vacca, W. D. 2005, ApJ, 626, 70

Ferrarese, L. \& Merritt, D. 2000, ApJL, 539, L9

Fischer, J. et al. 1999, Ap\&SS 266, 91

Fixsen, D. J., Dwek, E., Mather, J. C., Bennett, C. L., \& Shafer, R. A. 1998, ApJ, 508, 123

Flores, H., et al. 1999, ApJ, 517, 148

Fosbury, R. \& Wall, J. 1979, MNRAS, 189, 79

Fox, M. J., et al. 2002, MNRAS, 331, 839

Franceschini, A., et al. 2003a, MNRAS, 343, 1181

Franceschini, A., et al. 2003b, A\&A, 403, 501

Franceschini, A., et al. 2004, AJ, 129, 2074

Frayer, D. T., et al. 2004, ApJS, 154, 137

Gallagher, S. C., Brandt, W. N., Chartas, G., Garmire, G. P., \& Sambruna, R. M. 2002, ApJ, 569, 655

Gao, Y. \& Solomon, P. 2004, ApJ, 606, 271

Geach, J., et al. 2005, MNRAS, in press (astro-ph/0508357)

Gebhardt, K., et al. 2000, ApJ, 539, 13

Gehrz, R. D., Sramek, R. A. \& Weedman, D. W. 1983, ApJ, 267, 551

Genzel, R., et al. 1998, ApJ, 498, 579

Genzel, R., \& Cesarsky, C. J. 2000, ARA\&A, 38, 761

Genzel, R., Tacconi, L. J., Rigopoulou, D., Lutz, D., \& Tecza, M. 2001, ApJ, 563,527

Genzel, R., et al. 2003, ApJ, 584, 633

Goldader, J. D., et al. 2002, ApJ, 568, 651

González-Alfonso, E. Smith, H., Fischer, J. \& Cernicharo, J. 2004, ApJ, 613247

Granato, G. L., et al. 2000, ApJ, 542, 710

Granato, G. L., De Zotti, G., Silva, L., Bressan, A., \& Danese, L. 2004, ApJ, 600,580 
Greve, T. R., et al. 2004, MNRAS, 354, 779

Greve, T. R., et al. 2005, MNRAS, 359, 1165

Guiderdoni, B., Hivon, E., Bouchet, F. R., \& Maffei, B. 1998, MNRAS, 295, 877

Haas, M., et al. 2003, A\&A, 402, 87

Hacking, P., Houck, J. \& Condon, J. 1987, ApJL, 316, L15

Hatton, S., Devriendt, J. E. G., Ninin, S., Bouchet, F. R., Guiderdoni, B., \& Vibert, D. 2003, MNRAS, 343, 75

Hatziminaoglou, E. \& the SWIRE Team 2005, ApJ, 129, 119

Hauser, M. G., et al. 1998, ApJ, 508, 25

Hauser, M. G., \& Dwek, E. 2001, ARA\&A, 39, 249

Heckman, T. M., Armus L. \& Miley G. K. 1987, AJ, 93, 276

Heckman, T. M., Dahlem, M., Eales, S. A., Fabbiano, G., \& Weaver, K. 1996, ApJ, 457, 616

Heckman, T. M. 2001, in "Gas and Galaxy Evolution", ASP Conf 240, 345

Helou, G., Soifer, B. T., \& Rowan-Robinson, M. 1985, ApJL, 298, L7

Henkel, C., \& Wilson, T. L. 1990, A\&A, 229, 431

Hildebrand, R. H. 1983, QJRAS, 24, 267

Hines, D., Schmidt, G., Smith, P., Cutri, R. \& Low, F. 1995, ApJL, 450, L1

Holland, W. S., et al. 1999, MNRAS, 303, 659

Hopkins, P. F., et al. 2005a, ApJL, 625, L71

Hopkins, P. F., et al. 2005b, ApJ, 630, 705

Hopkins, P. F., et al. 2005c, ApJ, 630, 716

Hopkins, P. F., et al. 2005d, ApJ, 631, in press (astro-ph/0504253)

Hopkins, P. F., et al. 2005e, ApJ, in press (astro-ph/0506398)

Houck, J. R., Schneider, D., Danielson, G., Neugebauer, G., Soifer, B. T., Beichman, C. \& Lonsdale, C. J. 1985, ApJL, 290, L5

Houck, J. R., et al. 2005, ApJL, 622, L105

Hughes D. H., et al. 1998, Nature, 394, 241

Hutchings, J. B., \& Neff, S. G. 1991, AJ, 101, 434

Imanishi, M., Terashima, Y., Anabuki, N. \& Nakagawa, T. 2003, ApJ, 596L, 167

Ivison, R. J., et al. 2000, MNRAS, 315, 209

Ivison, R. J., et al. 2002, MNRAS, 337, 1

Ivison, R. J., et al. 2004, ApJS, 154, 124

Iwasawa, K., \& Comastri, A. 1998, MNRAS, 297, 1219

Iwasawa, K., Matt, G., Guainazzi, M., \& Fabian, A. C. 2001, MNRAS, 326, 894

Iwasawa, K., Sanders, D. B., Evans, A. S., Trentham, N., Miniutti, G., \& Spoon,

H. W. W. 2005, MNRAS, 357, 565

Joseph, R. D. 1999, Ap\&SS, 266, 321

Joseph, R. D. \& Wright G. S. 1985, MNRAS, 214, 87

Kandalyan, R. A. 1996, Astrophysics, 39, 237

Kandalyan, R. A. 2003, A\&A, 404, 513

Kashlinsky, A. 2005, Phys. Rep., 409, 361

Kauffmann, G., Colberg, J. M., Diaferio, A., \& White, S. D. M. 1999, MNRAS, 307,529

Kaviani, A., Haehnelt, M. G., \& Kauffmann, G. 2003, MNRAS, 340, 739

Kawada, M., et al. 1994, ApJL, 425, L89 
Kessler, M. F., et al. 1996, A\&A, 315, L27

Kewley, L. J., et al. 2000, ApJ, 530, 704

Kewley, L. J., Dopita, M. A., Sutherland, R., Heisler, C. A., \& Trevana, J. 2001, ApJ, 556, 121

Kim, D.-C. \& Sanders, D. B. 1998, ApJS, 119, 41

Kim, D.-C., Veilleux, S., \& Sanders, D. B. 1998, ApJ, 508, 627

Klaas, U., et al. 2001, A\&A, 379, 823

Kleinmann, D. E. \& Low, F. J. 1970, ApJL, 159, L165

Kleinmann, S. G., et al. 1988, ApJ, 328, 161

Klöckner, H., Baan, W. A., \& Garrett, M. A. 2003, Nature, 421, 821

Klöckner, H.-R., \& Baan, W. A. 2004, A\&A, 419, 887

Kobayashi, C. 2004, MNRAS, 347, 740

Komossa, S., Burwitz, V., Hasinger, G., Predehl, P., Kaastra, J. \& Ikebe, Y. 2005, ApJL, 582, L15

Kormendy, J. \& Sanders, D. B. 1992, ApJL, 390, L53

Kunth, D. \& Sargent, W. L. W. 1981, A\&A, 101, L5

Lagache, G., Puget, J. \& Dole, H. 2005, ARA\&A, 43

Larson, R. B. \& Tinsley, B. M. 1978, ApJ, 219, 46

Laurent, O., Mirabel, I. F., Charmandaris, V., Gallais, P., Madden, S. C., Sauvage, M., Vigroux, L., \& Cesarsky, C. 2000, A\&A, 359, 887

Laurent, G. T., et al. 2005, ApJ, 623, 742

Lawrence, A. 2001, MNRAS, 323, 147

Lawrence, A., Rowan-Robinson, M., Leech, K., Jones, D. H. P., \& Wall, J. V. 1989, MNRAS, 240, 329

Le Flóch, E., et al. 2005, ApJ, in press (astro-ph/0506462)

Leech, K. J., Rowan-Robinson, M., Lawrence, A., \& Hughes, J. D. 1994, MNRAS, 267,253

Leipski, C., et al. 2005, A\&A, 440, L5

Leitherer, C., et al. 1999, ApJS, 123, 3

Lemke, D., et al. 1996, A\&A, 315, L64

Lemonon, L., et al. 1998, A\&A, 334, L21

Liang, Y. C., Hammer, F., Flores, H., Elbaz, D., Marcillac, D., \& Cesarsky, C. J. 2004, A\&A, 423, 867

Lim, J., \& Ho, P. T. P. 1999, ApJL, 510, L7

Lipari, S. 1994, ApJ, 436, 102

Lipari, S., et al. 2003, MNRAS, 340, 289

Lo, K.-W. 2005, ARA\&A, 43

Lonsdale, C. J., Persson, S. E. \& Matthews, K. 1984, ApJ, 287, 95

Lonsdale, C. J., Hacking, P. B., Conrow, T. P. \& Rowan-Robinson, M. 1990, ApJ, 358, 60

Lonsdale, C. J., Smith, H. E., \& Lonsdale, C. J. 1993, ApJL, 405, L9

Lonsdale, C. J., Diamond, P. J., Lonsdale, C. J. \& Smith, H. E. 1994, Nature, 370,117

Lonsdale, C. J., Smith, H. E., \& Lonsdale, C. J. 1995, ApJ, 438, 632

Lonsdale, C. J., Lonsdale, C. J., Diamond, P. J., \& Smith, H. E. 1998, ApJL, 493, L13 
Lonsdale Carol J., Lonsdale Colin J., Smith, H. E., Diamond P. J. 2003a, ApJ, 592,804

Lonsdale, C. J., et al. 2003b, PASP, 115, 897

Lonsdale, C. J., Diamond, P. J., Lonsdale, C. J., Smith, H. E., Rovilos, E. 2006a, ApJ, in preparation

Lonsdale, C. J., Polletta, M., Farrah, D., et al. 2006, ApJ, submitted

Low, F. J. \& Kleinmann, D. E. 1968, AJ, 73, 868

Luhman, M. L., et al. 2003, ApJ, 594, 758

Lutz, D., Spoon, H. W. W., Rigopoulou, D., Moorwood, A. F. M., \& Genzel, R. 1998, ApJL, 505, L103

Lutz, D., Veilleux, S. \& Genzel, R. 1999, ApJL, 517, L13

Lutz, D., et al. 2005, ApJL, 625, L83

Lutz, D., et al. 2006, ApJL, in press (astro-ph/0509058)

MacKenty, J. \& Stockton, A. 1984, ApJ, 283, 64

Madau, P., Ferguson, H. C., Dickinson, M. E., Giavalisco, M., Steidel, C. C., \& Fruchter, A. 1996, MNRAS, 283, 1388

Magliocchetti, M., Moscardini, L., Panuzzo, P., Granato, G. L., De Zotti, G., \& Danese, L. 2001, MNRAS, 325, 1553

Malhotra, S., et al. 1997, ApJL, 491, L27

Malhotra, S., et al. 2001, ApJ, 561, 766

Maloney, P. R., \& Reynolds, C. S. 2000, ApJL, 545, L23

Martinez-Sansigre, A. et al. 2005, Nature, in press (astro-ph/0505486)

Martini, P. 2001, AJ, 121, 2301

Matarrese, S., Coles, P., Lucchin, F., \& Moscardini, L. 1997, MNRAS, 286, 115

McDowell, J. C., et al. 2003, ApJ, 591, 154

Melnick, J., \& Mirabel, I. F. 1990, A\&A, 231, L19

Meza, A., Navarro, J. F., Steinmetz, M., \& Eke, V. R. 2003, ApJ, 590, 619

Mihos, J. C. \& Hernquist, L. 1996, ApJ, 464, 641

Mirabel, I. F. \& Sanders, D. 1988, ApJ, 335, 104

Mirabel, I. F. 2002 in "Lighthouses of the Universe: The Most Luminous Celestial Objects and Their Use for Cosmology", p. 108

Miyoshi, M. et al. 1996, Nature, 373, 127

Mo, H. J., \& White, S. D. M. 2002, MNRAS, 336, 112

Momjian, E., Romney, J. D., Carilli, C. L., Troland, T. H., \& Taylor, G. B. 2003, ApJ, 587, 160

Morel, T., et al. 2001, MNRAS, 327, 1187

Moscardini, L., Coles, P., Lucchin, F., \& Matarrese, S. 1998, MNRAS, 299, 95

Murphy, T. W., et al. 1996, AJ, 111, 1025

Naab, T., \& Burkert, A. 2003, ApJ, 597, 893

Nagamine, K., Cen, R.-Y., Hernquist, L., Ostriker, J. \& Springel, V. 2005a, ApJ, 618, 23

Nagamine, K., Cen, R.-Y., Hernquist, L., Ostriker, J. \& Springel, V. 2005a, ApJ, 627, 608

Neff, S. G., Ulvestad, J. S., \& Teng, S. H. 2004, ApJ, 611, 186

Neri, R., et al. 2003, ApJL, 597, L113

Netzer, H., et al. 2005, ApJ, 629, 739 
Norris, R. P., Kesteven, M. J., Troup, E. R., Allen, D. A., \& Sramek, R. A. 1990, ApJ, 359, 291

Oliver, S. J., Rowan-Robinson, M., \& Saunders, W. 1992, MNRAS, 256, 15P

Oliver, S. J., et al. 1996, MNRAS, 280, 673

Oliver, S. J., et al. 1997, MNRAS, 289, 471

Oliver, S. J., et al. 2000, MNRAS, 316, 749

Oliver, S. J. \& Pozzi, F. 2005, to be published in 'ISO science legacy - a compact review of ISO major achievements', Space Science Reviews, Springer

Osterbrock, D. E., \& de Robertis, M. M. 1985, PASP, 97, 1129

Osterbrock, D. E., Tran, H. D., \& Veilleux, S. 1992, ApJ, 389, 196

Papovich, C., et al. 2004, ApJS, 154, 70

Partridge, R. B., \& Peebles, P. J. E. 1967, ApJ, 148, 377

Peeters, E., Spoon, H., \& Tielens, A. 2004, ApJ, 613, 986

Percival, W. J., Miller, L., McLure, R. J., \& Dunlop, J. S. 2001, MNRAS, 322, 843

Percival, W. J., Scott, D., Peacock, J. A., \& Dunlop, J. S. 2003, MNRAS, 338, L31

Pérez-González, P. G., et al. 2005, ApJ, 630, 82

Pierre, M., et al. 2001, A\&A, 372, L45

Pihlstrom, Y., Conway, J., Booth, R., Diamond, P. \& Polatidis, A. 2001, A\&A, 377, 413

Pihlstrom, Y. M., et al. 2005, ApJ, 618, 705

Polletta, M., Courvoisier, T., Hooper, E. \& Wilkes, B. 2000, A\&A, 362, 75

Polletta, M., et al. 2006, ApJ, submitted

Pope, A., Borys, C., Scott, D., Conselice, C., Dickinson, M., \& Mobasher, B. 2005, MNRAS, 358, 149

Pozzi, F., et al. 2004, ApJ, 609, 122

Ptak A., Heckman T., Levenson N. A., Weaver K. \& Strickland D. 2003, ApJ, 592, 782

Puget, J.-L., et al. 1996, A\&A, 308, L5

Puget, J. L., et al. 1999, A\&A, 345, 29

Readhead, A., Taylor, G., Pearson, T. \& Wilkinson, P. 1996, ApJ, 460, 634

Rees, M. J., Silk, J., Warner, M. W. \& Wickramasinghe, N. 1969, Nature, 223, 788

Rieke, G. H. \& Low F. J. 1972, ApJ, 176, L95

Rieke, G. H. \& Lebofsky, M. J. 1979, ARA\&A, 17, 477

Rieke, G. H. \& Lebofsky, M. J. 1986, ApJ, 304, 326

Rieke, G. H. 1988, ApJL, 331, L5

Rigopoulou, D., Spoon, H., Genzel, R., Lutz D., Moorwood, A. \& Tran Q. 1999, AJ, 118, 2625

Risaliti, G., Gilli, R., Maiolino, R. \& Salvati M. 2000, A\&A, 357, 13

Robertson, B., et al. 2005, ApJL, in press (astro-ph/0503369)

Rocca-Volmerange, B., Le Borgne, D., De Breuck, C., Fioc, M., \& Moy, E. 2004, A\&A, 415, 931

Rovilos, E., Diamond, P. J., Lonsdale, C. J., Lonsdale, C. J., \& Smith, H. E. 2003, MNRAS, 342, 373 
Rovilos, E., Diamond, P. J., Lonsdale, C. J., Smith, H. E., \& Lonsdale, C. J. 2005, MNRAS, 359, 827

Rowan-Robinson, M. \& Crawford, J. 1989, MNRAS, 238, 523

Rowan-Robinson, M., Hughes, J., Vedi, K., \& Walker, D. W. 1990, MNRAS, 246,273

Rowan-Robinson, M., et al. 1991, Nature, 351, 719

Rowan-Robinson, M., et al. 1997, MNRAS, 289, 490

Rowan-Robinson, M. 2000, MNRAS, 316, 885

Rowan-Robinson, M. 2001, ApJ, 549, 745

Rowan-Robinson, M., et al. 2004, MNRAS, 351, 1290

Rowan-Robinson, M., et al. 2005, AJ, 129, 1183

Rupke, D. S., Veilleux, S., \& Sanders, D. B. 2002, ApJ, 570, 588

Rupke, D. S., Veilleux, S., \& Sanders, D. B. 2005, ApJ, in press (astro-ph/0507037)

Sajina, A., et al. 2003, MNRAS, 343, 1365

Sakamoto, K., Scoville, N., Yun, M., Crosas, M., Genzel, R. \& Tacconi, L. 1999,ApJ, 514, 68

Sánchez, S. F., et al. 2004, ApJ, 614, 586

Sanders, D. et al. 1986, ApJL, 305, L45

Sanders, D. B., et al. 1988a, ApJ, 325, 74

Sanders, D. B., Soifer, B. T., Elias, J. H., Neugebauer, G. \& Matthews K. 1988b, ApJ, 328, L35

Sanders, D., Scoville, N. \& Soifer, B. T. 1991, ApJ, 370, 158

Sanders, D. B. \& Mirabel, I. F. 1996, ARA\&A, 34, 749

Satyapal, S., Sambruna, R. M., \& Dudik, R. P. 2004, A\&A, 414, 825

Saunders, W., et al. 1990, MNRAS, 242, 318

Schlegel, D. J., Finkbeiner, D. P., \& Davis, M. 1998, ApJ, 500, 525

Scoville, N., Yun, M. \& Bryant, P. 1997, ApJ, 484, 702

Scoville, N. Z., et al. 2000, AJ, 119, 991

Scott, S. E., et al. 2002, MNRAS, 331, 817

Shier, L. M., Rieke, M. J., \& Rieke, G. H. 1996, ApJ, 470, 222

Simpson, C., et al. 2004, MNRAS, 353, 179

Smail, I., Ivison, R. J., \& Blain, A. W. 1997, ApJL, 490, L5

Smail, I., Ivison, R. J., Blain, A. W., \& Kneib, J.-P. 1998, ApJL, 507, L21

Smail, I., et al. 1999, MNRAS, 308, 1061

Smail, I., Ivison, R. J., Blain, A. W., \& Kneib, J.-P. 2002, MNRAS, 331, 495

Smail, I., Scharf, C. A., Ivison, R. J., Stevens, J. A., Bower, R. G., \& Dunlop, J. S. 2003, ApJ, 599, 86

Smail, I., Chapman, S. C., Blain, A. W., \& Ivison, R. J. 2004, ApJ, 616, 71

Smith, H. E., Lonsdale, C. J., \& Lonsdale, C. J. 1998, ApJ, 492, 137

Smith, H. E., Lonsdale, C. J., Lonsdale, C. J. \& Diamond P. J. 1998, ApJL, 493, L17

Soifer, B. T., et al. 1984, ApJL, 278, L71

Soifer B. T., et al. 1984, ApJL, 283, L1

Soifer, B. T. \& Neugebauer, G. 1991, AJ, 101, 354

Soifer, B. T., et al. 1987, ApJ, 320, 238 
Soifer, B. T., et al. 1999, ApJ, 513, 207

Soifer, B. T., et al. 2000, AJ, 119, 509

Soifer, B. T., Neugebauer, G., Matthews, K., Egami, E., \& Weinberger, A. 2002, AJ, 124, 2980

Solomon, P., Downes, D. \& Radford, S. 1992, ApJL, 387, L55.

Solomon, P., Downes, D., Radford, S. \& Barrett, J. 1997, ApJ, 478, 144

Somerville, R. S., Primack, J. R., \& Faber, S. M. 2001, MNRAS, 320, 504

Spergel, D. N., et al. 2003, ApJS, 148, 175

Spinoglio, L., Andreani, P., \& Malkan, M. A. 2002, ApJ, 572, 105

Spoon, H. et al. 2002, A\&A, 385, 1022

Spoon, H. et al. 2004, ApJS, 154, 184

Springel, V., \& Hernquist, L. 2005, ApJL, 622, L9

Springel, V., Di Matteo, T. \& Hernquist, L. 2005, MNRAS, 361, 776

Stanford, S., Stern, D., van Breugel, W. \& De Brueck, C. 2000, ApJS, 131, 185

Stevens, J. A., et al. 2003, Nature, 425, 264

Strauss, M., Davis, M., Yahil, A., \& Huchra, J. 1990, ApJ, 361, 49

Sturm, E., et al. 2002, A\&A, 393, 821

Sugai, H., \& Malkan, M. A. 2000, ApJ, 529, 219

Surace, J. A., Sanders, D. B., Vacca, W. D., Veilleux, S. \& Mazzarella, J. M. 1998, ApJ, 492, 116

Surace, J. A., Sanders, D. B., \& Evans, A. S. 2000, ApJ, 529, 170

Surace, J. A., and the SWIRE Team 2005, ApJ, in press

Swinbank, A. M., Smail, I., Chapman, S. C., Blain, A. W., Ivison, R. J., \& Keel, W. C. 2004, ApJ, 617, 64

Swinbank, A. M., et al. 2005, MNRAS, 359, 401

Tacconi, L. J., et al. 2002, ApJ, 524, 732

Tacconi, L. J., et al. 2002, ApJ, 580, 73

Tamura, N., \& Ohta, K. 2003, AJ, 126, 596

Taylor, E., et al. 2005, MNRAS, 361, 1352

Tecza, M., et al. 2004, ApJL, 605, L109

Teng, S., et al. 2005, ApJ, in press (astro-ph/0508112)

Toomre, A., \& Toomre, J. 1972, ApJ, 178, 623

Tran Q. D., et al. 2001, ApJ, 552, 527

Turner, T., George, I., Nandra, K., \& Mushotzky, R. 1998, ApJ, 493, 91

Ulvestad, J. S., Wrobel, J. M., \& Carilli, C. L. 1999, ApJ, 516, 127

Vaccari, M. et al. 2005, MNRAS, 358,397

van Breugel, W., Fragile, C., Anninos, P. \& Murray, S. 2004, IAU Symp, 271, 472

van der Kruit, P. C. 1971, A\&A, 15, 110

van Dokkum, P. G., Franx, M., Fabricant, D., Kelson, D. D., \& Illingworth, G. D. 1999, ApJL, 520, L95

van Gorkum, J. \& Hibbard, 2005, ARA\&A, 43

van Kampen, E., Jimenez, R., \& Peacock, J. A. 1999, MNRAS, 310, 43

van Kampen, E., et al. 2005, MNRAS, 359, 469

Vanden Bout, P. \& Solomon, P. 2005, ARA\&A, 43

Veilleux, S., \& Osterbrock, D. E. 1987, ApJS, 63, 295 
Veilleux, S., Kim, D.-C., Sanders, D. B., Mazzarella, J. M., \& Soifer, B. T. 1995, ApJS, 98, 171

Veilleux, S., Sanders, D. B. \& Kim D.-C. 1997, ApJ, 484, 92

Veilleux, S., Kim, D.-C., \& Sanders, D. B. 1999a, ApJ, 522, 113

Veilleux, S., Sanders, D. B., \& Kim, D.-C. 1999b, ApJ, 522, 139

Veilleux, S., Kim, D.-C. \& Sanders, D. B. 2002, ApJS, 143, 315

Veilleux, S., Cecil, \& Bland-Hawthorn, J. 2005, ARA\&A, 43

Verma, A., Rowan-Robinson, M., McMahon, R. \& Efstathiou, A. 2002, MNRAS, 335,574

Verma, A., et al. 2005, to be published in 'ISO science legacy - a compact review of ISO major achievements', Space Science Reviews, Springer

Vignati, P., et al. 1999, A\&A, 349, L57

Voit, G. M., Weymann, R. J. \& Koirsta, K. T. 1993. ApJ, 413, 95

Webb, T. M., et al. 2003, ApJ, 597, 680

Weiler, K. W., Panagia, N., \& Sramek, R. A. 1990, ApJ, 364, 611

Wilman, R. J., Fabian, A. C., Cutri, R. M., Crawford, C. S., \& Brandt, W. N. 1998, MNRAS, 300, L7

Wilman, R. J., Crawford, C. S., \& Abraham, R. G. 1999, MNRAS, 309, 299

Wilman, R. J., Fabian, A. C., Crawford, C. S., \& Cutri, R. M. 2003, MNRAS, 338, L19

Wisotzki, L., et al. 2000, A\&A, 358, 77

Wold, M., Lacy, M., Lilje, P. B., \& Serjeant, S. 2001, MNRAS, 323, 231

Wold, M., Armus, L., Neugebauer, G., Jarrett, T. H., \& Lehnert, M. D. 2003, AJ, 126,1776

Worsley, M.A. et al. 2005, MNRAS, 357, 1281

Xia, X. Y., Xue, S. J., Mao, S., Boller, T., Deng, Z. G., \& Wu, H. 2002, ApJ, 564,196

Xu, C. K., Lonsdale, C. J., Shupe, D. L., O’Linger, J. \& Masci, F. 2001, ApJ, 562,179

Xu, C. K., Lonsdale, C. J., Shupe, D. L., Franceschini, A., Martin, C., \& Schiminovich, D. 2003, ApJ, 587, 90

Yan, L., et al. 2005, ApJ, 628, 604

Yun, M. S. \& Scoville, N. Z. 1998, ApJ, 507, 774

Yun, M. S., Reddy, N. A., \& Condon, J. J. 2001, ApJ, 554, 803

Zou, Z., Xia, X., Deng, Z., \& Su, H. 1991, MNRAS, 252, 593 